\title{
Comparison of Approximation Methods for Computing Tolerance Factors for a Multivariate Normal Population
}

\section{K. KRISHNAMOORTHY}

Department of Mathematics

University of Southwestern Louisiana

Lafayette, LA 70504

(kxk4695@usl.edu)

\section{Thomas MATHEW}

Department of Mathematics and Statistics University of Maryland Baltimore County

Baltimore, MD 21250

(mathew@research.umbc.edu)

\begin{abstract}
In this article, we compare several approximation methods for computing the tolerance factors of a multivariate normal population. These approximate methods are evaluated by comparing the Monte Carlo estimates of the coverage probabilities with those of the specified ones. Numerical studies indicate that, in general, the tolerance factors based on an approximate method given by John, which is commonly used in the literature, are inaccurate when the number of variates is greater than or equal to 2, but another approximation (which is also due to John) with slight modification gives better results. Using John's idea, we also suggest some new approximations, which give satisfactory results. Two of the new approximations emerge as satisfactory candidates for practical use. Our fairly extensive numerical results provide guidelines regarding the choice of the tolerance factor for practical applications.
\end{abstract}

KEY WORDS: Chi-squared approximation; Noncentral chi-squared distribution; Wishart distribution.

\section{INTRODUCTION}

\subsection{Background}

Tolerance regions are used widely in industrial applications. The theory of tolerance regions is well developed in the univariate case, particularly for the univariate normal distribution; see Guttman (1970a) for a detailed treatment of the theory. For the construction of tolerance factors for a univariate normal distribution, tables, approximations, and programs are available; see Guttman (1970a, chap. 4) and Eberhardt, Mee, and Reeve (1989). The literature on the multivariate setup is rather limited, however. The first attempt at constructing tolerance regions for a multivariate normal distribution, applicable to finite samples, appears to be due to John (1963). He considered the setup in which the multivariate normal distribution has unknown mean and unknown variance-covariance matrix. John's (1963) work is quite significant because he not only developed the theoretical framework for the problem but also provided simple and easy-to-use approximations for computing the tolerance factors. Furthermore, he outlined a general approach for obtaining various approximations for the tolerance factors. An approach for deriving approximate tolerance factors was also described by Siotani (1964). The computations requircd to obtain his tolerance factor is rather involved, however, and he gave a practical solution only for the bivariate case. Related results were given by Chew (1966), who also reviewed the results of John (1963). It should be pointed out that early work due to Wald (1942) provided a large-sample procedure for computing tolerance regions. Other large-sample results were given by Guttman (1970b). In fact, when the sample size is 100 or more, Guttman (1970a, chap. 4) provided tables of tolerance factors for multivariate normal distributions of dimensions 2, 3, and 4 .

For the multivariate normal distribution, the computation of the tolerance factors involved the distribution of the eigenvalues of a Wishart matrix. John (1963) suggested approximations based on the distribution of the arithmetic mean, geometric mean, and harmonic mean of the eigenvalues. We shall describe these in Section 2. Note that, for the multivariate normal distribution, the tolerance factors can be cstimatcd by Montc Carlo. Indeed, for the bivariate normal distribution, Hall and Sheldon (1979) reported such results in the bivariate case. Fuchs and Kenett (1987, 1988), however, used the arithmetic-mean approximation suggested by John (1963). The approximate tolerance factor involves the percentiles of both central and noncentral chi-squared distributions. It appears that some confusion has resulted from John's unconventional notation for the noncentrality parameter of a noncentral chi-squared distribution; see Remark 2.1 in Section 2. Consequently, the tolerance factors given by Fuchs and Kenett $(1987,1988)$ are incorrect.

\subsection{Some Examples}

Several authors have applied tolerance regions to specific practical problems, assuming multivariate normality. Hall and Sheldon (1979) discussed an application dealing with ballistic miss distances. Fuchs and Kenett (1987) applied John's (1963) results to two examples, one dealing with testing adulteration in citrus juice (in which the data are six-

(c) 1999 American Statistical Association and the American Society for Quality TECHNOMETRICS, AUGUST 1999, VOL. 41, NO. 3 
dimensional) and a second example dealing with the diagnosis of atopic diseases based on the levels of immunoglobulin in blood (in which the data are three-dimensional). In the citrus-juice example, the six-dimensional data consisted of measurements of six attributes-total soluble solids produced at $20^{\circ} \mathrm{C}$, acidity, total sugars, potassium, formol number, and total pectin. The reference sample consisted of 80 specimens of pure juice. Once the tolerance region is computed, we can check if the measurement on an additional specimen to be tested falls in the tolerance region to decide whether or not it is adulterated.

In a later article, Fuchs and Kenett (1988) used multivariate tolerance regions in a quality-control application for deciding whether ceramic substrate plates used in the microelectronics industry conform to an accepted standard. The five-dimensional data used for this purpose consisted of measurements of physical dimensions of individual substrates. A tolerance region for this problem is a region that covers a specified proportion of the target standard population, with a certain confidence. Decision regarding conformity of the new substrates to the accepted standard is based on whether the corresponding five-dimensional observations belong to the tolerance region. Details of the problem, including the manufacturing steps, were described by Fuchs and Kenett (1988).

\subsection{Statement of the Problem, Summary, and Conclusions}

The major goal of this article is to investigate whether the various approximations for the tolerance factors derived for the multivariate normal distribution are satisfactory. We also have suggested some new approximations, one of which is based on the harmonic mean of the eigenvalues of a Wishart matrix, already mentioned by John (1963). His chi-squared approximation for the distribution of the harmonic mean appears to be unsatisfactory, however, as will be clear from the numerical results in Section 4. We have suggested a more satisfactory approximation by equating the first and second moments of the reciprocal of the harmonic mean of the eigenvalues of a Wishart matrix to the first and second moments of a scalar multiple of the reciprocal of a chi-squared random variable, which we call the modified harmonic-mean approximation. As the numerical results indicate, such an approximation is much more satisfactory, compared to the arithmetic-mean, geometric-mean, and harmonic-mean approximations of John (1963).

The article is organized as follows. In Section 2, we briefly discuss the theoretical derivation of the tolerance region. This was essentially given by John (1963). In Section 3 , we discuss the various approximations for computing the tolerance factor. The existing approximations are given and some new ones are proposed - the modified harmonic-mean approximation mentioned previously, as well as two other approximations using scalar-valued functions of a Wishart matrix instead of scalar-valued functions of its eigenvalues. Section 4 gives the numerical results for comparing the various approximations. The numerical results are reported for dimensions 2, 3, and 6, and for coverage and confidence levels $.90, .95$, and .99 . For the purpose of comparison, we have also included the tolerance factor, obtained by Monte Carlo. Our comparison of the tolerance factors is based on the simulated confidence coefficients of the various approximate tolerance factors.

Our conclusions are as follows. The tolerance factors based on the arithmetic-mean and geometric-mean approximations are very unsatisfactory. The corresponding tolerance factors are much smaller than the actual tolerance factor. Consequently, the corresponding confidence level is much smaller than the confidence level we choose. The situation becomes worse as the dimension gets large. The harmonic-mean approximation of John (1963) is slightly better, but it is still very unsatisfactory for practical use. The modified harmonic-mean approximation appears to provide comparatively better performance. The simulated confidence level of the resulting tolerance factor is also below the actual confidence level, however, the difference being somewhat significant in higher dimensions. In the bivariate case, we have also included Siotani's (1964) approximate tolerance factor in our numerical results. This approximation provides a conservative tolerance factor. That is, the corresponding confidence level is larger than the confidence level we choose. Moreover, Siotani's (1964) approximation is tractable only in the bivariate case. It turns out that our new approximations, based on scalar-valued functions of a Wishart matrix, are more satisfactory. One of the new approximations results in a tolerance factor that is somewhat large compared to the actual tolerance factor. In other words, the corresponding tolerance region is conservative. Our second approximation turns out to be much more satisfactory. Our approximations result in tolerance factors having explicit analytic expressions. In other words, they do not present any computational difficulty. Furthermore, our numerical results show the accuracy of the various approximations and provide some guidelines regarding the approximation to be used for practical purposes. Note that, for estimating the tolerance factor by Monte Carlo simulation, the parameters are the sample size, the dimension, coverage of the tolerance region, and the confidence level. Among our approximate tolerance factors, there is no single choice that is completely satisfactory for all values of the preceding parameters. In fact, our approximations are somewhat unsatisfactory when the coverage and the confidence level become large, especially in higher dimensions. In this situation, estimating the tolerance factor by Monte Carlo simulation appears to be the only option currently available. We have provided such estimated tolerance factors in Table 4 , Section 4 , for some selected values of the sample size and the dimension.

\section{OUTLINE OF THE THEORY}

Let $N_{p}(\mu, \Sigma)$ denote the $p$-variate normal distribution with mean vector $\mu$ and variance-covariance matrix $\Sigma$. Here both $\mu$ and $\Sigma$ are unknown. Let $\mathbf{x}_{1}, \mathbf{x}_{2}, \ldots, \mathbf{x}_{n}$ denote a random sample from $N_{p}(\mu, \Sigma)$, where $n>p$. Write

$$
\overline{\mathbf{x}}=\frac{1}{n} \sum_{i=1}^{n} \mathbf{x}_{i}, \quad A=\sum_{i=1}^{n}\left(\mathbf{x}_{i}-\overline{\mathbf{x}}\right)\left(\mathbf{x}_{i}-\overline{\mathbf{x}}\right)^{\prime}
$$


Then $\overline{\mathbf{x}}$ and $(1 / n) A$ are the maximum likelihood estimators of $\mu$ and $\Sigma$ and, furthermore, $(1 / n-1) A$ is an unbiased estimator of $\Sigma$; see Anderson (1984, chap. 3). Let x denote a future observation from $N_{p}(\mu, \Sigma)$, where $\mathbf{x}$ is independent of $\mathbf{x}_{1}, \mathbf{x}_{2}, \ldots, \mathbf{x}_{n}$. $\Lambda$ tolerance region is a region for $\mathbf{x}$ satisfying the inequality

$$
(n-1)(\mathbf{x}-\overline{\mathbf{x}})^{\prime} A^{-1}(\mathbf{x}-\overline{\mathbf{x}}) \leq c,
$$

where $c$ is the tolerance factor, to be determined subject to the condition that the region in (2.2) contains at least a proportion $\beta$ of the normal distribution $N_{p}(\mu, \Sigma)$, with confidence $\gamma$. Specifically, $c$ satisfies

$$
P_{\overline{\mathbf{x}}, A}\left[P_{\mathbf{x}}\left\{(n-1)(\mathbf{x}-\overline{\mathbf{x}})^{\prime} A^{-1}(\mathbf{x}-\overline{\mathbf{x}}) \leq c \mid \overline{\mathbf{x}}, A\right\} \geq \beta\right]=\gamma .
$$

The main problem is the computation of the tolerance factor $c$ satisfying (2.3). An explicit analytic form for $c$ is not available. Thus $c$ must be computed either numerically using Monte Carlo methods or by using a satisfactory approximation. In the bivariate case (i.e., $p=2$ ), Hall and Sheldon (1979) provided tables of the the tolerance factors obtained by Monte Carlo methods for various values of $n, \beta$, and $\gamma$. Such a computation can be quite time consuming, especially in higher dimensions. Hence, it is desirable to derive good approximations. John (1963) suggested some approximations, and his main result is stated in the following theorem. We shall use the following notation. Let

$$
V=\Sigma^{-1 / 2} A \Sigma^{-1 / 2},
$$

and let $l_{1}>\cdots>l_{p}>0$ be the ordered eigenvalues of $V$. Write $1=\left(l_{1}, l_{2}, \ldots, l_{p}\right)^{\prime}$. Note that $V \sim W_{p}\left(I_{p}, n-1\right)$, the $p$-dimensional Wishart distribution with $\mathrm{df}=n-1$ and associated scale matrix $I_{p}$.

Theorem 2.1. Suppose that 1 is as defined previously, and let $\xi(\mathbf{l})$ be a real valued function of 1 such that $l_{p}<\xi(1)<l_{1}$. Furthermore, let $\chi^{2}(\alpha ; m, \delta)$ denote the $100 \alpha$ th percentile of a noncentral chi-squared distribution with $\mathrm{df}=m$ and noncentrality parameter $\delta$, and let $\nu_{2}$ denote the $100(1-\gamma)$ th percentile of $\xi(1)$. Then, an approximate expression for $c$ that satisfies (2.3) is given by

$$
c=(n-1) \chi^{2}(\beta ; p, p / n) / \nu_{2} .
$$

John (1963) justified the approximation for large values of $n$. For completeness, we have given a proof of Theorem 2.1 in Appendix A. Note that the value of $c$ in (2.5) depends on the $\xi(\mathbf{l})$ that we choose. Several choices are discussed in Section 3 .

Remark 2.1. John (1963) used the notation $\delta / 2$ for the noncentrality parameter of a noncentral chi-squared distribution, which we denote by $\delta$ in the statement of Theorem 2.1. Our notation appears to be the standard notation; see also Chew (1966, sec. 5.2). Thus, in John's notation, (2.5) will become $c=(n-1) \chi^{2}(\beta ; p, p / 2 n) / \nu_{2}$. This difference in notation appears to have caused some confusion in the literature, especially in the work of Fuchs and Kenett (1987, 1988).

\section{APPROXIMATE TOLERANCE FACTORS}

We shall now give several approximate tolerance factors, based on different choices of the quantity $\xi(1)$ in Theorem 2.1. The arithmetic-mean, geometric-mean, and harmonicmean approximations described here were given by John (1963). In the rest of the article, we shall use the following notation. The central chi-squared distribution with $\mathrm{df}$ $=m$ will be denoted by $\chi^{2}(m)$ and its $100 \alpha$ th percentile by $\chi^{2}(\alpha ; m)$. The noncentral chi-squared distribution with $\mathrm{df}=m$ and noncentrality parameter $\delta$ will be denoted by $\chi^{2}(m, \delta)$ and its $100 \alpha$ th percentile by $\chi^{2}(\alpha ; m, \delta)$.

\subsection{The Arithmetic-Mean Approximation}

Let

$$
\xi(\mathbf{l})=\frac{\sum_{i=1}^{p} l_{i}}{p}=\frac{\operatorname{tr}(V)}{p}=\frac{\operatorname{tr}\left(A \Sigma^{-1}\right)}{p},
$$

where $V$ is given in (2.4). Because $\operatorname{tr}(V)=\operatorname{tr}\left(A \Sigma^{-1}\right) \sim$ $\chi^{2}((n-1) p), \nu_{2}$, the $100(1-\gamma)$ th percentile of $\operatorname{tr}\left(A \Sigma^{-1}\right) /$ $p$, is given by

$$
\nu_{2}=\frac{\chi^{2}(1-\gamma ;(n-1) p)}{p} .
$$

From (2.5), the approximate tolerance factor, say $c_{a m}$, is given by

$$
c_{\mathrm{am}}=\frac{p(n-1) \chi^{2}(\beta ; p, p / n)}{\chi^{2}(1-\gamma ;(n-1) p)} .
$$

\subsection{The Geometric-Mean Approximation}

Choose

$$
\xi(\mathbf{l})=\left\{\prod_{i=1}^{p} l_{i}\right\}^{1 / p}=|V|^{1 / p}=\left|A \Sigma^{-1}\right|^{1 / p} .
$$

Then it is known that $\xi(\mathbf{l})$ is approximately distributed as a multiple of a gamma random variable with shape parameter $p(n-p) / 2$. The approximate distribution is given by

$$
\frac{g_{1}^{p(n-p) / 2} y^{p(n-p) / 2-1} e^{-g_{1} y}}{\Gamma\left(\frac{p(n-p)}{2}\right)},
$$

where $y=\xi(\mathbf{l})$ and

$$
g_{1}=\frac{p}{2}\left[1-\frac{(p-1)(p-2)}{2 n}\right]^{1 / p} .
$$

The preceding approximate distribution of $\xi(\mathbf{l})$ is due to Hoel (1937) and was also given by Anderson (1984, p. 265) and John (1963, p. 366). [There are two typographical errors in John's expression (5.5) for $g_{1}$; the exponent $1 / p$ is missing and the denominator should be $2 n$, not $2(n-1)$.] Hence, if we choose $\xi(1)$ as the geometric mean given in (3.4), $\nu_{2}$ in (2.5) is approximately equal to $\left(1 / g_{1}\right) G(1-\gamma ; p(n-p) / 2)$, where $g_{1}$ is given in (3.5) and $G(1-\gamma ; p(n-p) / 2)$ denotes the $100(1-\gamma)$ th percentile of the gamma distribution with 
Table 1. Tolerance Factors and the Corresponding Confidence Levels When $p=2$

\begin{tabular}{|c|c|c|c|c|c|c|c|c|c|c|}
\hline$n$ & $\beta$ & $\gamma$ & $c_{a m}$ & $c_{g m}$ & $c_{h m}$ & $c_{m h m}$ & $c_{V}$ & $c_{V h m}$ & $c_{\text {siot }}$ & $c_{s i m}^{*}$ \\
\hline \multirow[t]{18}{*}{10} & .90 & .90 & 8.39 & 9.78 & 11.70 & 12.25 & 13.05 & 12.51 & 15.00 & 12.82 \\
\hline & & & .715 & .803 & .877 & .891 & .910 & .898 & .942 & .904 \\
\hline & & .95 & 9.70 & 11.44 & 13.87 & 14.94 & 16.67 & 15.47 & 19.59 & 16.08 \\
\hline & & & .810 & .873 & .930 & .945 & .964 & .951 & .977 & .961 \\
\hline & & .99 & 12.99 & 15.67 & 19.55 & 22.38 & 27.67 & 23.90 & 35.92 & 25.86 \\
\hline & & & .913 & .946 & .965 & .984 & .996 & .975 & .988 & .979 \\
\hline & .95 & .90 & 10.89 & 12.71 & 15.19 & 15.91 & 16.96 & 16.25 & 19.49 & 16.79 \\
\hline & & & .673 & .778 & .859 & .878 & .897 & .881 & .933 & .895 \\
\hline & & .95 & 12.60 & 14.87 & 18.01 & 19.40 & 21.66 & 20.10 & 25.45 & 22.19 \\
\hline & & & .768 & .848 & .913 & .929 & .947 & .936 & .976 & .950 \\
\hline & & .99 & 16.87 & 20.36 & 25.40 & 29.07 & 35.94 & 31.05 & 46.66 & 36.66 \\
\hline & & & .890 & .938 & .966 & .979 & .989 & .983 & .999 & .991 \\
\hline & .99 & .90 & 16.69 & 19.48 & 23.29 & 24.39 & 25.99 & 24.91 & 29.87 & 28.46 \\
\hline & & & .632 & .732 & .818 & .858 & .868 & .842 & .916 & .893 \\
\hline & & .95 & 19.32 & 22.78 & 27.61 & 29.74 & 33.19 & 30.80 & 39.00 & 35.77 \\
\hline & & & .735 & .825 & .898 & .912 & .935 & .921 & .960 & .950 \\
\hline & & .99 & 25.86 & 31.21 & 38.92 & 44.55 & 55.08 & 47.59 & 71.51 & 61.14 \\
\hline & & & .874 & .928 & .964 & .987 & .987 & .978 & .999 & .994 \\
\hline \multirow[t]{18}{*}{20} & .90 & .90 & 6.72 & 7.16 & 7.67 & 7.79 & 8.45 & 7.98 & 8.49 & 7.94 \\
\hline & & & .771 & .829 & .890 & .891 & .943 & .908 & .947 & .908 \\
\hline & & .95 & 7.38 & 7.90 & 8.48 & 8.67 & 9.78 & 8.99 & 9.68 & 8.97 \\
\hline & & & .862 & .902 & .935 & .939 & .973 & .952 & .969 & .951 \\
\hline & & .99 & 8.88 & 9.55 & 10.33 & 10.70 & 13.09 & 11.35 & 12.81 & 11.24 \\
\hline & & & .952 & .970 & .983 & .989 & .999 & .993 & .998 & .993 \\
\hline & .95 & .90 & 8.74 & 9.32 & 9.97 & 10.13 & 10.99 & 10.38 & 11.05 & 10.48 \\
\hline & & & .762 & .825 & .873 & .887 & .927 & .893 & .932 & .900 \\
\hline & & .95 & 9.60 & 10.27 & 11.03 & 11.27 & 12.72 & 11.69 & 12.59 & 11.81 \\
\hline & & & .840 & .889 & .928 & .932 & .964 & .946 & .964 & .947 \\
\hline & & .99 & 11.55 & 12.42 & 13.43 & 13.91 & 17.03 & 14.76 & 16.67 & 15.35 \\
\hline & & & .935 & .960 & .978 & .980 & .998 & .987 & .997 & .990 \\
\hline & .99 & .90 & 13.42 & 14.31 & 15.32 & 15.55 & 16.89 & 15.95 & 16.97 & 16.99 \\
\hline & & & .703 & .778 & .826 & .841 & .896 & .853 & .897 & .897 \\
\hline & & .95 & 14.75 & 15.77 & 16.94 & 17.31 & 19.54 & 17.95 & 19.33 & 19.18 \\
\hline & & & .776 & .822 & .882 & .901 & .958 & .921 & .953 & .945 \\
\hline & & .99 & 17.73 & 19.08 & 20.63 & 21.37 & 26.16 & 22.68 & 25.60 & 25.26 \\
\hline & & & .902 & .935 & .959 & .971 & .995 & .978 & .992 & $\begin{array}{r}.988 \\
\text { (continued) }\end{array}$ \\
\hline
\end{tabular}

shape parameter $p(n-p) / 2$. This gives the following approximate tolerance factor, denoted by $c_{\mathrm{gm}}$ :

$$
c_{\mathrm{gm}}=\frac{g_{1}(n-1) \chi^{2}(\beta ; p, p / n)}{G\left(1-\gamma ; \frac{p(n-p)}{2}\right)} .
$$

\subsection{The Harmonic-Mean Approximation}

Let

$$
\xi(\mathbf{l})=p / \sum_{i=1}^{p} \frac{1}{l_{i}}=p / \operatorname{tr}\left(V^{-1}\right)=p / \operatorname{tr}\left(A^{-1} \Sigma\right) .
$$

With $\xi(1)$ as given in (3.7), John (1963) approximated the distribution of $p \xi(1)$ with a chi-squared distribution with $\mathrm{df}$ $=(n-1) p-p(p+1)+2$. Using the percentile of such a chisquared distribution gives the following tolerance factor, denoted by $c_{\mathrm{hm}}$ :

$$
c_{\mathrm{hm}}=\frac{p(n-1) \chi^{2}(\beta ; p, p / n)}{\chi^{2}(1-\gamma ;(n-1) p-p(p+1)+2)} .
$$

The following justification can be given for choosing $\xi(\mathbf{l})$ as the harmonic mean given in (3.7). Let $\mathbf{z}=$ $\left(z_{1}, z_{2}, \ldots, z_{p}\right)^{\prime} \sim N_{p}\left(0, I_{p}\right)$ and $\mathbf{q}=\left(q_{1}, q_{2}, \ldots, q_{p}\right)^{\prime} \sim$ $N_{p}\left(0,(1 / n) I_{p}\right)$, where $\mathbf{z}$ and $\mathbf{q}$ are independently dis- tributed. From the proof of Theorem 2.1, given in Appendix $\mathrm{A}$, it follows that $\xi(1)$ is to be chosen so as to have the following approximation [see (A.6) in Appendix A]:

$$
\begin{aligned}
P_{\mathbf{z}}\left[(n-1) \sum_{i=1}^{p}\right. & \left.\left(z_{i}-q_{i}\right)^{2} / l_{i} \leq c \mid \mathbf{q}, \mathbf{l}\right] \\
& \doteq P_{\mathbf{z}}\left[(n-1) \sum_{i=1}^{p}\left(z_{i}-q_{i}\right)^{2} / \xi(\mathbf{l}) \leq c \mid \mathbf{q}, \mathbf{l}\right] .
\end{aligned}
$$

Clearly, the preceding approximation will hold if the following approximation is satisfactory:

$$
\begin{aligned}
(\mathbf{z}-\mathbf{q})^{\prime} \operatorname{diag}\left(\frac{1}{l_{1}}, \frac{1}{l_{2}}, \ldots, \frac{1}{l_{p}}\right)(\mathbf{z}-\mathbf{q}) & \\
& \doteq \frac{1}{\xi(\mathbf{l})}(\mathbf{z}-\mathbf{q})^{\prime}(\mathbf{z}-\mathbf{q}) .
\end{aligned}
$$

ln other words, we need to compute $\xi(\mathbf{l})$ so that $\operatorname{diag}\left(1 / l_{1}, 1 / l_{2}, \ldots, 1 / l_{p}\right)$ can be approximated using $(1 /$ $\xi(1)) I_{p}$. An obvious way of computing such a $\xi(\mathbf{l})$ is to minimize

$$
\operatorname{tr}\left[\left(\operatorname{diag}\left(\frac{1}{l_{1}}, \frac{1}{l_{2}}, \ldots, \frac{1}{l_{p}}\right)-\frac{1}{\xi(\mathbf{I})} I_{p}\right)^{2}\right] .
$$

TECHNOMETRICS, AUGUST 1999, VOL. 41, NO. 3 
Table 1. (continued)

\begin{tabular}{|c|c|c|c|c|c|c|c|c|c|c|}
\hline$n$ & $\beta$ & $\gamma$ & $C_{a m}$ & $c_{g m}$ & $c_{h m}$ & $c_{m h m}$ & $c_{V}$ & $c_{V h m}$ & $c_{\text {siot }}$ & $c_{s i m}^{*}$ \\
\hline \multirow[t]{18}{*}{30} & .90 & .90 & 6.17 & 6.43 & 6.70 & 6.75 & 7.29 & 6.91 & 7.13 & 6.83 \\
\hline & & & .812 & .858 & .902 & .907 & .955 & .927 & .924 & .913 \\
\hline & & .95 & 6.65 & 6.93 & 7.24 & 7.32 & 8.15 & 7.56 & 7.82 & 7.43 \\
\hline & & & .868 & .902 & .933 & .947 & .970 & .952 & .961 & .947 \\
\hline & & .99 & 7.68 & 8.03 & 8.42 & 8.56 & 10.17 & 9.00 & 9.48 & 8.71 \\
\hline & & & .960 & .976 & .985 & .988 & .998 & .996 & .997 & .993 \\
\hline & .95 & .90 & 8.03 & 8.36 & 8.72 & 8.79 & 9.48 & 8.99 & 9.27 & 9.00 \\
\hline & & & .778 & .830 & .871 & .878 & .931 & .897 & .917 & .898 \\
\hline & & .95 & 8.65 & 9.02 & 9.42 & 9.52 & 10.60 & 9.83 & 10.17 & 9.87 \\
\hline & & & .861 & .896 & .925 & .934 & .973 & .946 & .963 & .950 \\
\hline & & .99 & 10.00 & 10.45 & 10.95 & 11.14 & 13.23 & 11.71 & 12.33 & 11.62 \\
\hline & & & .957 & .967 & .975 & .983 & .998 & .984 & .999 & .983 \\
\hline & .99 & .90 & 12.34 & 12.85 & 13.39 & 13.50 & 14.56 & 13.81 & 14.25 & 14.34 \\
\hline & & & .752 & .803 & .853 & .848 & .913 & .873 & .888 & .902 \\
\hline & & .95 & 13.30 & 13.86 & 14.47 & 14.63 & 16.29 & 15.10 & 15.63 & 15.65 \\
\hline & & & .820 & .855 & .885 & .913 & .952 & .910 & .939 & .939 \\
\hline & & .99 & 15.36 & 16.06 & 16.82 & 17.12 & 20.33 & 17.99 & 18.94 & 19.09 \\
\hline & & & .923 & .948 & .966 & .973 & .991 & .981 & .989 & .989 \\
\hline \multirow[t]{18}{*}{40} & .90 & .90 & 5.89 & 6.07 & 6.25 & 6.28 & 6.73 & 6.41 & 6.53 & 6.30 \\
\hline & & & .793 & .834 & .878 & .881 & .943 & .907 & .933 & .888 \\
\hline & & .95 & 6.28 & 6.47 & 6.67 & 6.72 & 7.40 & 6.91 & 7.03 & 6.80 \\
\hline & & & .909 & .937 & .947 & .949 & .987 & .967 & .968 & .961 \\
\hline & & .99 & 7.09 & 7.32 & 7.57 & 7.65 & 8.90 & 7.99 & 8.18 & 7.98 \\
\hline & & & .969 & .979 & .984 & .986 & .998 & .995 & .996 & .995 \\
\hline & .95 & .90 & 7.66 & 7.89 & 8.13 & 8.17 & 8.76 & 8.34 & 8.49 & 8.39 \\
\hline & & & .808 & .843 & .865 & .878 & .934 & .894 & .922 & .901 \\
\hline & & .95 & 8.17 & 8.41 & 8.68 & 8.74 & 9.62 & 8.99 & 9.15 & 9.02 \\
\hline & & & .879 & .911 & .938 & .982 & .975 & .956 & .970 & .963 \\
\hline & & .99 & 9.23 & 9.52 & 9.85 & 9.95 & 11.57 & 10.39 & 10.64 & 10.27 \\
\hline & & & .962 & .974 & .980 & .984 & .997 & .988 & .991 & .986 \\
\hline & .99 & .90 & 11.78 & 12.13 & 12.50 & 12.56 & 13.46 & 12.82 & 13.05 & 13.13 \\
\hline & & & .762 & .798 & .838 & .851 & .908 & .872 & .888 & .892 \\
\hline & & .95 & 12.55 & 12.93 & 13.34 & 13.43 & 14.79 & 13.81 & 14.06 & 14.20 \\
\hline & & & .829 & .872 & .902 & .917 & .968 & .935 & .942 & .950 \\
\hline & & .99 & 14.18 & 14.64 & 15.13 & 15.30 & 17.79 & 15.97 & 16.35 & 16.33 \\
\hline & & & .946 & .959 & .973 & .978 & .996 & .982 & .987 & .986 \\
\hline
\end{tabular}

NOTE: The contidence levels appear below the tolerance factors.

${ }^{*} c_{\text {sim }}$ denotes the tolerance factor estimated by Monte Carlo simulation.

This is equivalent to minimizing $\sum_{i=1}^{p}\left(\left(1 / l_{i}\right)-(1 / \xi(1))\right)^{2}$, and the solution is easily seen to be the harmonic mean given in (3.7).

\subsection{A Modified Harmonic-Mean Approximation}

We shall once again consider $\xi(1)=p / \operatorname{tr}\left(V^{-1}\right)$ given in (3.7). Instead of John's (1963) chi-squared approximation of the distribution of $p \xi(1)$, we shall consider approximating the distribution of $\operatorname{tr}\left(V^{-1}\right)$ by that of $a / \chi^{2}(b)$, where the constants $a$ and $b$ will be determined by equating the first and second moments of $\operatorname{tr}\left(V^{-1}\right)$ to those of $a / \chi^{2}(b)$. Toward this, we note that

$$
\begin{aligned}
E\left[\operatorname{tr}\left(V^{-1}\right)\right] & =p /(n-p-2) \\
E\left[\left\{\operatorname{tr}\left(V^{-1}\right)\right\}^{2}\right] & -\frac{p[(n-p-3) p+2]}{(n-p-1)(n-p-2)(n-p-4)} .
\end{aligned}
$$

The expression for $E\left[\operatorname{tr}\left(V^{-1}\right)\right]$ is immediately obvious from that of $E\left[V^{-1}\right]$ given, for example, by Anderson (1984, p. 270). The expression for $E\left[\left\{\operatorname{tr}\left(V^{-1}\right)\right\}^{2}\right]$ can be obtained from von Rosen [1988, corollary 3.1 (v)]. Moreover,

$$
\begin{aligned}
E\left(a / \chi^{2}(b)\right) & =\frac{a}{(b-2)} \\
E\left[\left(a / \chi^{2}(b)\right)^{2}\right] & =\frac{a^{2}}{(b-2)(b-4)} .
\end{aligned}
$$

Equating the first two moments in (3.9) to those in (3.10), we get

$$
\begin{aligned}
& b=\frac{p(n-p-1)(n-p-4)+4(n-2)}{n-2} \\
& \text { and } a=\frac{p(b-2)}{n-p-2} .
\end{aligned}
$$

Thus, $\nu_{2}$, the $100(1-\gamma)$ th percentile of $\xi(\mathbf{l})$ in (3.7), can be approximated by the $100(1-\gamma)$ th percentile of $p \chi^{2}(b) / a$, where $a$ and $b$ are given in (3.11). This gives the following approximate tolerance factor, denoted by $c_{\mathrm{mhm}}$ :

$$
c_{\mathrm{mhm}}=\frac{a(n-1) \chi^{2}(\beta ; p, p / n)}{p \chi^{2}(1-\gamma ; b)} .
$$

\subsection{An Approximation Based on $v_{11.2}$}

Recall that $\xi(\mathbf{l})$ satisfies $l_{p} \leq \xi(\mathbf{l}) \leq l_{1}$. We shall now consider a function of $V$, say $\xi(V)$, that satisfies $l_{p} \leq \xi(V) \leq l_{1}$, where $\xi(V)$ is not a function of 1 . The choice that we 
Table 2. Tolerance Factors and the Corresponding Confidence Levels When $p=3$

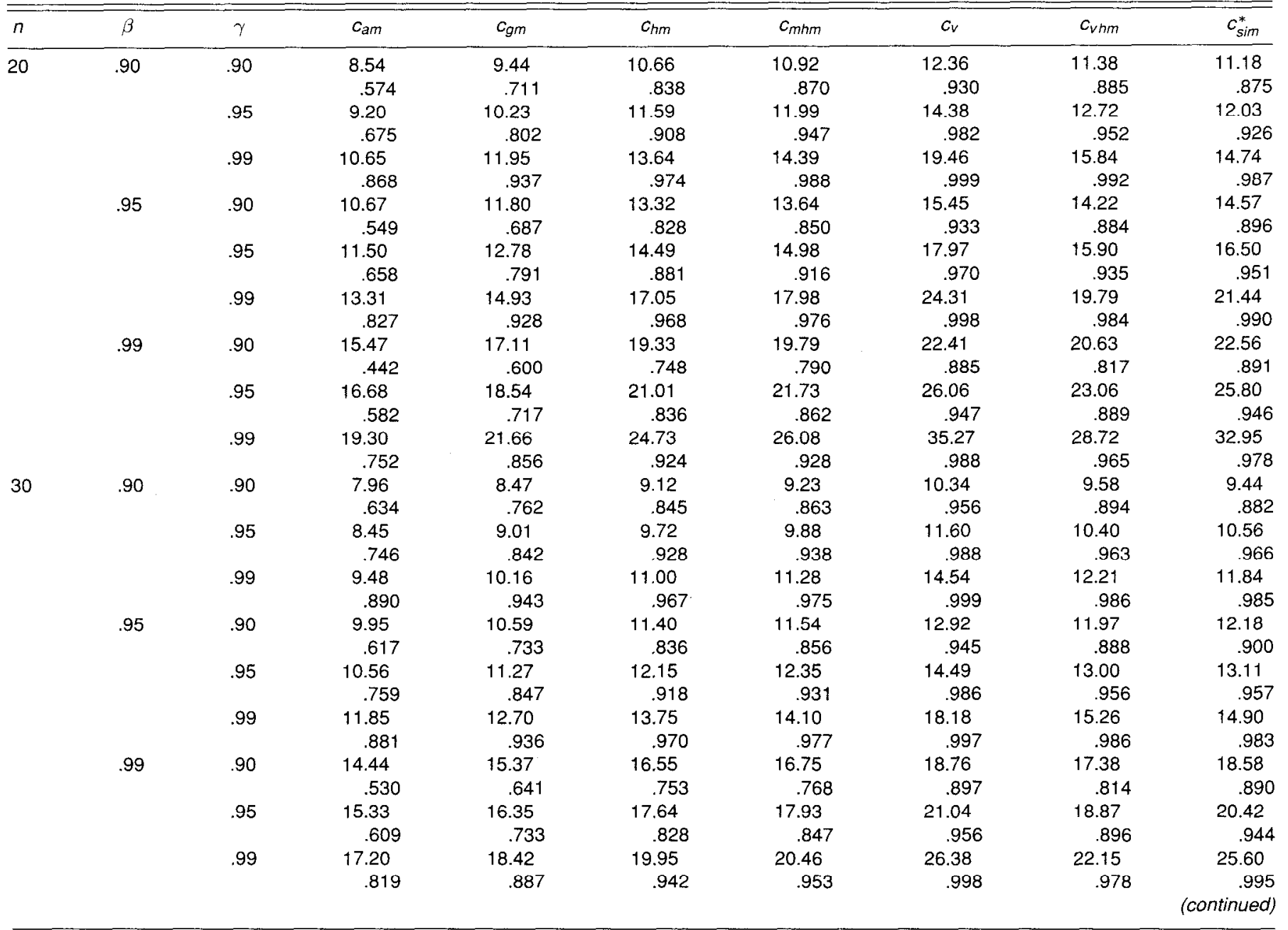

shall make is

$$
\xi(V)=v_{11.2},
$$

where $v_{11.2}^{-1}$ is the first element of $V^{-1}$. More explicitly, suppose that $V$ is partitioned as

$$
V=\left(\begin{array}{ll}
v_{11} & \mathbf{v}_{12} \\
\mathbf{v}_{21} & V_{22}
\end{array}\right)
$$

where $v_{11}$ is a scalar, $\mathbf{v}_{21}$ is a $(p-1) \times 1$ vector, $\mathbf{v}_{12}=\mathbf{v}_{21}^{\prime}$, and $V_{22}$ is $(p-1) \times(p-1)$. Then $v_{11.2}=v_{11}-\mathbf{v}_{12} V_{22}^{-1} \mathbf{v}_{21}$. Because any diagonal element of a positive definite matrix is less than or equal to the largest eigenvalue, we get $v_{11.2} \leq$ $v_{11} \leq l_{1}$. Furthermore, because $v_{11.2}^{-1}$ is the first diagonal element of $V^{-1}$ and because $1 / l_{p}$ is the largest eigenvalue of $V^{-1}$, we also have $v_{11.2}^{-1} \leq 1 / l_{p}$; that is, $v_{11.2} \geq l_{p}$. In other words, $l_{p} \leq v_{11.2} \leq l_{1}$. Because $v_{11.2} \sim \chi^{2}(n-p)$ (see Anderson 1984, theorem 7.3.6), the choice $\xi(V)=v_{11.2}$ gives the following approximate tolerance factor, denoted by $c_{n}$ :

$$
c_{v}=\frac{(n-1) \chi^{2}(\beta ; p, p / n)}{\chi^{2}(1-\gamma ; n-p)} .
$$

Remark 3.1. The use of $v_{11.2}$ may appear somewhat arbitrary, because $v_{11.2}$ is not a symmetric function of $V$. Note, however, that $v_{11.2}$ is not directly used for computing the tolerance factor in (3.14). We use its distribution only to obtain the percentile $\nu_{2}$ for computing the tolerance factor in (2.5). The $100(1-\gamma)$ th percentile of any random variable lying between $l_{p}$ and $l_{1}$ is a potential choice for $\nu_{2}$. The validity of the resulting tolerance factor can then be investigated numerically.

\subsection{An Approximation Based on the Harmonic Mean and $v_{11.2}$}

Our numerical results in Section 4 show that, in general, the tolerance factor $c_{\mathrm{mhm}}$ in (3.12) is somewhat smaller than the actual tolerance factor, whereas $c_{v}$ in (3.15) is somewhat large. This suggests that a tolerance factor between $c_{\mathrm{mhm}}$ and $c_{v}$ may be quite satisfactory. Recall that $c_{\mathrm{mhm}}$ is derived based on the choice $\xi(\mathbf{l})=p / \operatorname{tr}\left(V^{-1}\right)$ and $c_{v}$ is derived based on $\xi(V)=v_{11.2}$. We shall now consider a choice of $\xi(V)$ between $p / \operatorname{tr}\left(V^{-1}\right)$ and $v_{11.2}$. The choice that we shall make is the harmonic mean of $p / \operatorname{tr}\left(V^{-1}\right)$ and $v_{11.2}$. Specifically, let

$$
\xi(V)=2 /\left[\frac{\operatorname{tr}\left(V^{-1}\right)}{p}+v_{11.2}^{-1}\right] .
$$

The main reason for choosing the harmonic mean (as opposed to the arithmetic mean or the geometric mean) is that we can easily approximate the distribution of $1 / \xi(V)$ 
Table 2. (continued)

\begin{tabular}{|c|c|c|c|c|c|c|c|c|c|}
\hline$n$ & $\beta$ & $\gamma$ & $c_{a m}$ & $c_{g m}$ & $c_{h m}$ & $c_{m n m}$ & $c_{V}$ & $c_{v h m}$ & $c_{\text {sim }}^{*}$ \\
\hline \multirow[t]{18}{*}{40} & .90 & .90 & 7.66 & 8.01 & 8.45 & 8.51 & 9.43 & 8.80 & 8.57 \\
\hline & & & .692 & .800 & .898 & .904 & .973 & .938 & .915 \\
\hline & & .95 & 8.06 & 8.44 & 8.91 & 9.00 & 10.38 & 9.42 & 9.11 \\
\hline & & & .794 & .868 & .932 & .938 & .992 & .967 & .947 \\
\hline & & .99 & 8.89 & 9.33 & 9.87 & 10.03 & 12.52 & 10.75 & 10.28 \\
\hline & & & .932 & .964 & .984 & .988 & .999 & .995 & 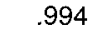 \\
\hline & .95 & .90 & 9.57 & 10.01 & 10.56 & 10.64 & 11.79 & 10.99 & 11.02 \\
\hline & & & .639 & .745 & .841 & .830 & .946 & .891 & .893 \\
\hline & & .95 & 10.07 & 10.55 & 11.14 & 11.25 & 12.97 & 11.77 & 11.77 \\
\hline & & & .785 & .868 & .925 & .931 & .991 & .963 & .963 \\
\hline & & .99 & 11.11 & 11.66 & 12.34 & 12.53 & 15.65 & 13.43 & 13.29 \\
\hline & & & .913 & .952 & .974 & .981 & .999 & .995 & .993 \\
\hline & .99 & .90 & 13.90 & 14.53 & 15.33 & 15.44 & 17.11 & 15.96 & 16.48 \\
\hline & & & .565 & .665 & .757 & .792 & .908 & .813 & .878 \\
\hline & & .95 & 14.62 & 15.31 & 16.17 & 16.33 & 18.83 & 17.08 & 17.64 \\
\hline & & & .665 & .748 & .853 & .865 & .970 & .899 & .935 \\
\hline & & .99 & 16.12 & 16.93 & 17.91 & 18.19 & 22.71 & 19.50 & 20.32 \\
\hline & & & .828 & .889 & .940 & .952 & .998 & .974 & .987 \\
\hline \multirow[t]{18}{*}{50} & .90 & .90 & 7.47 & 7.73 & 8.06 & 8.10 & 8.91 & 8.35 & 8.17 \\
\hline & & & .733 & .811 & .888 & .889 & .971 & .927 & .899 \\
\hline & & .95 & 7.81 & 8.10 & 8.45 & 8.51 & 9.68 & 8.86 & 8.67 \\
\hline & & & .813 & .876 & .924 & .929 & .989 & .965 & .947 \\
\hline & & .99 & 8.52 & 8.84 & 9.24 & 9.34 & 11.40 & 9.93 & 9.75 \\
\hline & & & .932 & .957 & .978 & .988 & .999 & .994 & .992 \\
\hline & .95 & .90 & 9.34 & 9.67 & 10.08 & 10.13 & 11.13 & 10.44 & 10.41 \\
\hline & & & .693 & .783 & .863 & .855 & .966 & .907 & .904 \\
\hline & & .95 & 9.77 & 10.12 & 10.56 & 10.63 & 12.10 & 11.08 & 11.07 \\
\hline & & & .799 & .858 & .911 & .921 & .989 & .955 & .954 \\
\hline & & .99 & 10.65 & 11.05 & 11.55 & 11.67 & 14.24 & 12.42 & 12.21 \\
\hline & & & .923 & .951 & .974 & .976 & .999 & .993 & .991 \\
\hline & .99 & .90 & 13.55 & 14.03 & 14.63 & 14.70 & 16.16 & 15.15 & 15.65 \\
\hline & & & .606 & .697 & .771 & .777 & .913 & .832 & .883 \\
\hline & & .95 & 14.17 & 14.69 & 15.33 & 15.43 & 17.57 & 16.08 & 16.23 \\
\hline & & & .692 & .772 & .849 & .873 & .967 & .909 & .916 \\
\hline & & .99 & 15.45 & 16.05 & 16.76 & 16.94 & 20.68 & 18.02 & 18.41 \\
\hline & & & .875 & .918 & .943 & .951 & .997 & .977 & .983 \\
\hline
\end{tabular}

NOTE: The confidence levels appear below the tolerance factors

${ }^{*} c_{\text {sim }}$ denotes the tolerance factor estimated by Monte Carlo simulation.

with that of $d / \chi^{2}(e)$, where $d$ and $e$ are obtained by equating the first two moments. Recall that this approach was used to arrive at $c_{\mathrm{mhm}}$ in (3.12). The first two moments of $1 / \xi(V)$ are derived in Appendix B [see (B.4) and (B.9)], and the first two moments of $d / \chi^{2}(e)$ are similar to those in (3.10). Equating the two sets of moments, we get

$$
\begin{array}{r}
e=\frac{4 p(n-p-1)(n-p)-12(p-1)(n-p-2)}{3(n-2)+p(n-p-1)}, \\
d=(e-2) /(n-p-2) .
\end{array}
$$

Thus, $\nu_{2}$, the $100(1-\gamma)$ th percentile of $\xi(V)$ in (3.16), can be approximated by the $100(1-\gamma)$ th percentile of $\chi^{2}(e) / d$, where $d$ and $e$ are as given in (3.17). The resulting approximate tolerance factor will be denoted by $c_{v \mathrm{hm}}$ and is given by

$$
c_{v \mathrm{hm}}=\frac{d(n-1) \chi^{2}(\beta ; p, p / n)}{\chi^{2}(1-\gamma ; e)} .
$$

Remark 3.2. When $p=1$, all the approximate tolerance factors given previously coincide, and the common value, say $c_{1}$, is given by

$$
c_{1}=\frac{(n-1) \chi^{2}(\beta, 1,1 / n)}{\chi^{2}(1-\gamma ; n-1)} .
$$

Furthermore, numerical studies indicate that the approximation $c_{1}$ in (3.19) is as good as Wald and Wolfowitz's (1946) approximation for the univariate case. (See also Kendall and Stuart 1973, p. 133.) When $p \geq 2$, however, we will see that these approximations are quite different.

\subsection{Siotani's Approximation}

Siotani (1964) derived an approximate tolerance factor by choosing $\nu_{2}$ to be the $100(1-\gamma)$ th percentile of the quantity

$$
\begin{aligned}
h(\mathrm{l}) & =\left(\prod_{i=1}^{p} l_{i}\right)^{1 / p}\left[\frac{\left(\prod_{i=1}^{p} l_{i}\right)^{1 / p}}{\sum_{i=1}^{p} l_{i} / p}\right]^{2} \\
& =|V|^{1 / p}\left[\frac{|V|^{1 / p}}{\operatorname{tr}(V) / p}\right]^{2} ;
\end{aligned}
$$

see equation (42) of Siotani (1964). Because $|V|^{1 / p} \leq$ $\operatorname{tr}(V) / p$ and because $|V|^{1 / p} \leq l_{1}$, it follows that $h(\mathbf{l}) \leq l_{1}$. It is not true, however, that $h(\mathbf{l}) \geq l_{p}$. (For example, if $p=2, l_{1}=1$, and $l_{2}=.0004$, the inequality $h(\mathrm{I}) \geq l_{2}$ does 
Table 3. Tolerance Factors and the Corresponding Confidence Levels When $p=6$

\begin{tabular}{|c|c|c|c|c|c|c|c|c|c|}
\hline$n$ & $\beta$ & $\gamma$ & $c_{a m}$ & $c_{g m}$ & $c_{h m}$ & $c_{m h m}$ & $c_{v}$ & $c_{V h m}$ & $c_{\text {sim }}^{*}$ \\
\hline \multirow[t]{18}{*}{30} & .90 & .90 & 12.71 & 14.57 & 16.86 & 17.21 & 20.37 & 18.31 & 18.16 \\
\hline & & & .159 & .482 & .803 & .827 & .977 & .905 & .894 \\
\hline & & .95 & 13.24 & 15.25 & 17.68 & 18.18 & 23.03 & 19.79 & 19.40 \\
\hline & & & .223 & .590 & .879 & .901 & .998 & .971 & .963 \\
\hline & & .99 & 14.33 & 16.64 & 19.36 & 20.19 & 29.38 & 23.01 & 22.23 \\
\hline & & & .393 & .783 & .948 & .958 & .999 & .994 & .989 \\
\hline & .95 & .90 & 15.03 & 17.24 & 19.94 & 20.36 & 24.09 & 21.66 & 22.28 \\
\hline & & & .118 & .353 & .712 & .752 & .946 & .856 & .893 \\
\hline & & .95 & 15.66 & 18.04 & 20.91 & 21.50 & 27.24 & 23.41 & 23.56 \\
\hline & & & .158 & .507 & .807 & .859 & .984 & .915 & .918 \\
\hline & & .99 & 16.95 & 19.68 & 22.89 & 23.88 & 34.75 & 27.22 & 28.37 \\
\hline & & & .358 & .701 & .910 & .941 & .999 & .987 & .990 \\
\hline & .99 & .90 & 20.07 & 23.01 & 26.62 & 27.18 & 32.16 & 28.91 & 32.37 \\
\hline & & & .064 & .227 & .568 & .628 & .875 & .745 & .881 \\
\hline & & .95 & 20.91 & 24.08 & 27.91 & 28.70 & 36.36 & 31.25 & 35.06 \\
\hline & & & .099 & .326 & .640 & .710 & .954 & .830 & .934 \\
\hline & & .99 & 22.62 & 26.27 & 30.56 & 31.88 & 46.38 & 36.34 & 40.80 \\
\hline & & & .234 & .553 & .824 & .864 & .997 & .956 & .990 \\
\hline \multirow[t]{18}{*}{40} & .90 & .90 & 12.35 & 13.63 & 15.09 & 15.27 & 17.76 & 16.13 & 15.87 \\
\hline & & & .211 & .523 & .803 & .829 & .981 & .916 & .892 \\
\hline & & .95 & 12.79 & 14.15 & 15.68 & 15.94 & 19.64 & 17.17 & 16.57 \\
\hline & & & .318 & .642 & .885 & .897 & .995 & .969 & .949 \\
\hline & & .99 & 13.68 & 15.21 & 16.89 & 17.31 & 23.92 & 19.37 & 18.29 \\
\hline & & & .549 & .838 & .966 & .978 & 1.00 & .996 & .987 \\
\hline & .95 & .90 & 14.61 & 16.12 & 17.84 & 18.06 & 21.01 & 19.08 & 19.18 \\
\hline & & & .179 & .452 & .742 & .778 & .956 & .864 & .872 \\
\hline & & .95 & 15.13 & 16.74 & 18.55 & 18.86 & 23.23 & 20.31 & 20.09 \\
\hline & & & .256 & .553 & .821 & .852 & .997 & .949 & .933 \\
\hline & & .99 & 16.18 & 17.99 & 19.98 & 20.47 & 28.29 & 22.91 & 22.89 \\
\hline & & & .471 & .786 & .936 & .959 & 1.00 & .986 & .985 \\
\hline & .99 & .90 & 19.50 & 21.52 & 23.82 & 24.11 & 28.05 & 25.47 & 27.59 \\
\hline & & & .113 & .323 & .598 & .650 & .898 & .746 & .885 \\
\hline & & .95 & 20.20 & 22.35 & 24.76 & 25.17 & 31.01 & 27.11 & 28.99 \\
\hline & & & .181 & .428 & .692 & .735 & .962 & .838 & .915 \\
\hline & & .99 & 21.60 & 24.02 & 26.67 & 27.33 & 37.77 & 30.59 & 33.41 \\
\hline & & & .329 & .644 & .843 & .878 & .997 & .963 & $\begin{array}{r}.988 \\
\text { (continued) }\end{array}$ \\
\hline
\end{tabular}

not hold.) If $h_{\gamma}$ denotes the $100(1-\gamma)$ th percentile of $h(\mathbf{l})$, the tolerance factor suggested by Siotani (1964), say $c_{\text {siot }}$, is given by

$$
c_{\text {siot }}=\frac{(n-1) \chi^{2}(\beta ; p, p / n)}{h_{\gamma}}
$$

We would like to point out that in Siotani's (1964) article, the tolerance factor appears as in (3.21) without the quantity $(n-1)$ in the numerator; see his equation (46). The reason for this is that he defined $h(\mathbf{l})$ as in (3.20) using the eigenvalues of $V /(n-1)$, instead of those of $V$ as we have done. It is easy to verify that $h(l / n, 1)=$ $(1 / n-1) h(\mathrm{l})$.

The major hurdle in using (3.21) is the difficulty in computing $h_{\gamma}$. In the bivariate case, Siotani (1964) gave the density of $h(\mathbf{l})$ and also its cumulative distribution function, which can be used for the computation of $h_{\gamma}$. If $p \geq 3$, it appears that $h_{\gamma}$ has to be numerically obtained. When $p=2$, Siotani (1964) showed that

$$
\begin{aligned}
& P\left(h(\mathbf{l}) \leq h_{0}\right)=I_{h_{0}}(n-1) \\
& \quad+\frac{\Gamma[(2 n-1) / 3]}{\Gamma(n-1)} h_{0}^{(n-2) / 3}\left\{1-I_{h_{0}}((2 n-1) / 3)\right\},
\end{aligned}
$$

where $I_{c}(d)$ denotes the incomplete gamma function; see Equation (57) of Siotani (1964). To compute $c_{\text {siot }}$ in (3.21), the quantity $h_{\gamma}$ that we need is the value of $h_{0}$ that will make (3.22) equal to $1-\gamma$.

\section{MONTE CARLO STUDY}

We now evaluate the approximations given in Section 3 by comparing the Monte Carlo estimates $\hat{\gamma}$ of the coverage probabilities with the specified values of $\gamma$ for various values of $p$ and $n$. To compute the approximations, we used the IMSL FORTRAN subroutines CSNIN to compute the percentiles of a noncentral chi-squared distribution, CHIIN to compute the percentiles of a central chi-squared distribution, and GAMIN to compute the percentiles of a gamma distribution. Furthermore, we used the IMSL subroutine RNMVN to generate normal variates and the FORTRAN subroutine due to Smith and Hocking (1972) to generate Wishart variates.

In the Monte Carlo simulation study, we used the following expression, which is equivalent to (2.3) [see (A.1) in Appendix A]:

$$
P_{\mathbf{u}, V}\left[P_{\mathbf{y}}\left[(n-1)(\mathbf{y}-\mathbf{u})^{\prime} V^{-1}(\mathbf{y}-\mathbf{u}) \leq c \mid \mathbf{u}, V\right] \geq \beta\right]=\gamma,
$$


Table 3. (continued)

\begin{tabular}{|c|c|c|c|c|c|c|c|c|c|}
\hline$n$ & $\beta$ & $\gamma$ & $c_{a m}$ & $c_{g m}$ & $c_{h m}$ & $c_{m h m}$ & $c_{v}$ & $c_{v h m}$ & $c_{s i m}^{*}$ \\
\hline \multirow[t]{18}{*}{50} & .90 & .90 & 12.12 & 13.09 & 14.15 & 14.27 & 16.38 & 14.99 & 14.75 \\
\hline & & & .304 & .577 & .836 & .840 & .985 & .939 & .911 \\
\hline & & .95 & 12.50 & 13.53 & 14.64 & 14.80 & 17.86 & 15.81 & 15.22 \\
\hline & & & .393 & .682 & .888 & .909 & .999 & .971 & .938 \\
\hline & & .99 & 13.27 & 14.40 & 15.61 & 15.86 & 21.15 & 17.53 & 16.73 \\
\hline & & & .590 & .820 & .950 & .986 & .999 & .997 & .990 \\
\hline & .95 & .90 & 14.33 & 15.48 & 16.74 & 16.88 & 19.37 & 17.73 & 18.04 \\
\hline & & & .221 & .489 & .768 & .772 & .978 & .898 & .924 \\
\hline & & .95 & 14.79 & 16.00 & 17.31 & 17.50 & 21.13 & 18.71 & 18.70 \\
\hline & & & .346 & .644 & .859 & .893 & .998 & .964 & .964 \\
\hline & & .99 & 15.70 & 17.04 & 18.46 & 18.76 & 25.02 & 20.73 & 20.34 \\
\hline & & & .564 & .819 & .955 & .957 & .999 & .993 & .991 \\
\hline & .99 & .90 & 19.14 & 20.67 & 22.35 & 22.53 & 25.86 & 23.67 & 24.96 \\
\hline & & & .158 & .346 & .625 & .657 & .937 & .773 & .877 \\
\hline & & .95 & 19.75 & 21.36 & 23.11 & 23.37 & 28.20 & 24.97 & 26.40 \\
\hline & & & .222 & .455 & .721 & .742 & .980 & .864 & .945 \\
\hline & & .99 & 20.96 & 22.75 & 24.65 & 25.05 & 33.41 & 27.68 & 29.73 \\
\hline & & & 411 & .641 & .833 & .887 & .998 & .965 & .989 \\
\hline \multirow[t]{18}{*}{70} & .90 & .90 & 11.84 & 12.48 & 13.17 & 13.23 & 14.90 & 13.80 & 13.53 \\
\hline & & & .373 & .610 & .808 & .841 & .988 & .918 & .881 \\
\hline & & .95 & 12.15 & 12.83 & 13.54 & 13.62 & 15.99 & 14.41 & 13.79 \\
\hline & & & .493 & .715 & .879 & .926 & .997 & .972 & .919 \\
\hline & & .99 & 12.77 & 13.51 & 14.27 & 14.40 & 18.33 & 15.65 & 14.68 \\
\hline & & & .711 & .901 & .962 & .977 & 1.00 & .994 & .975 \\
\hline & .95 & .90 & 14.00 & 14.77 & 15.58 & 15.65 & 17.62 & 16.33 & 16.11 \\
\hline & & & .326 & .579 & .789 & .808 & .980 & .899 & .880 \\
\hline & & .95 & 14.37 & 15.17 & 16.01 & 16.11 & 18.91 & 17.04 & 16.82 \\
\hline & & & .467 & .704 & .879 & .871 & .995 & .961 & .952 \\
\hline & & .99 & 15.11 & 15.98 & 16.87 & 17.03 & 21.68 & 18.51 & 18.27 \\
\hline & & & .684 & .848 & .944 & .957 & 1.00 & .995 & .993 \\
\hline & .99 & .90 & 18.70 & 19.72 & 20.80 & 20.90 & 23.53 & 21.80 & 22.59 \\
\hline & & & .258 & .439 & .656 & .666 & .944 & .803 & .883 \\
\hline & & .95 & 19.19 & 20.26 & 21.38 & 21.51 & 25.25 & 22.76 & 23.55 \\
\hline & & & .313 & .524 & .737 & .747 & .979 & .882 & .937 \\
\hline & & .99 & 20.17 & 21.33 & 22.53 & 22.74 & 28.94 & 24.71 & 25.16 \\
\hline & & & .502 & .709 & .874 & .902 & .998 & .984 & .993 \\
\hline
\end{tabular}

NOTE: The confidence levels appear below the tolerance factors.

${ }^{*} c_{\text {sim }}$ denotes the tolerance factor estimated by Monte Carlo simulation.

where $\mathbf{y}, \mathbf{u}$, and $V$ are independent with $\mathbf{y} \sim N_{p}\left(0, I_{p}\right), \mathbf{u} \sim$ $N_{p}\left(0,(1 / n) I_{p}\right)$, and $V \sim W_{p}\left(I_{p}, n-1\right)$. Write $V=T T^{\prime}$, where $T$ is the lower triangular matrix with positive diagonal elements. For our simulation study, we need only $T$; Smith and Hocking's algorithm returns $T$ as one of the outputs. The simulation study was conducted as follows:

1 . For given $n, p, \beta$, and $\gamma$, compute an approximate value of $c$; call it $c_{0}$.

2. $i=1$; generate a $\mathbf{u}$ and a $T$; compute $T^{-1}$.

3. Generate $1,200 \mathbf{y}^{\prime}$ s and compute $\mathbf{a}^{\prime}=(\mathbf{y}-\mathbf{u})^{\prime \prime} T^{\prime-1}$ and the quadratic form $(n-1) \mathbf{a}^{\prime} \mathbf{a}$ for each $\mathbf{y}$. Find the proportion of the quadratic forms less than or equal to $c_{o}$. If this proportion is greater than or equal to $\beta$, then set $Z_{i}=1$; else $Z_{i}=0$.

4. Find the $100 \beta$ th percentile of the quadratic forms in step 3; call it $Q_{i}$.

5. $i=i+1$; go to Step 2 until $i=1,200$.

6. $\hat{\gamma}-\sum_{\imath=1}^{1,200} Z_{i} / 1,200$ is a Monte Carlo estimate of $\gamma$; $100 \gamma$ th percentile of the $Q_{i}$ 's is a Monte Carlo estimate of the tolerance factor $c$.

Note that it is not possible to estimate the tolerance factor and its coverage probability in a single simulation. A separate simulation is needed to estimate the coverage probabil- ity. To understand the accuracy of the simulation estimates, see the work of Hall and Sheldon (1979), who discussed the accuracy of the simulation estimates of the tolerance factors based on 1,200 by 1,200 runs for the bivariate case. For $n=10(10) 40, p=2$, and the values of $\gamma$ and $\beta$ that are of practical interest, we computed all seven approximations given in Section 3 and the corresponding Monte Carlo coverage probability estimates $\hat{\gamma}$. These values are given in Table 1 (pp. 237-238). For the purpose of comparison, we have also included the Monte Carlo estimate of the tolerance factor. For $p=3$ and $p=6$, we have carried out the same computation for all the approximations except Siotani's (1964). It was omitted because the cumulative distribution function (3.18) is applicable only in the bivariate case. Hence, the quantity $h_{\gamma}$ [required to compute $c_{\text {siot }}$ in (3.21)] has to be numerically obtained. We did not carry out this calculation. The results for $p=3$ and $p=6$ are given, respectively, in Table 2 and Table 3 (pp. 239-242).

The conclusions we draw from Tables 1-3 are the following. The arithmetic-mean approximation is the worst, followed by the geometric-mean and harmonic-mean approximations. The tolerance factors based on these approximations are much smaller than the actual tolerance factors, and hence the confidence level is also substantially less. The 
Table 4. Monte Carlo Estimates of Tolerance Factors

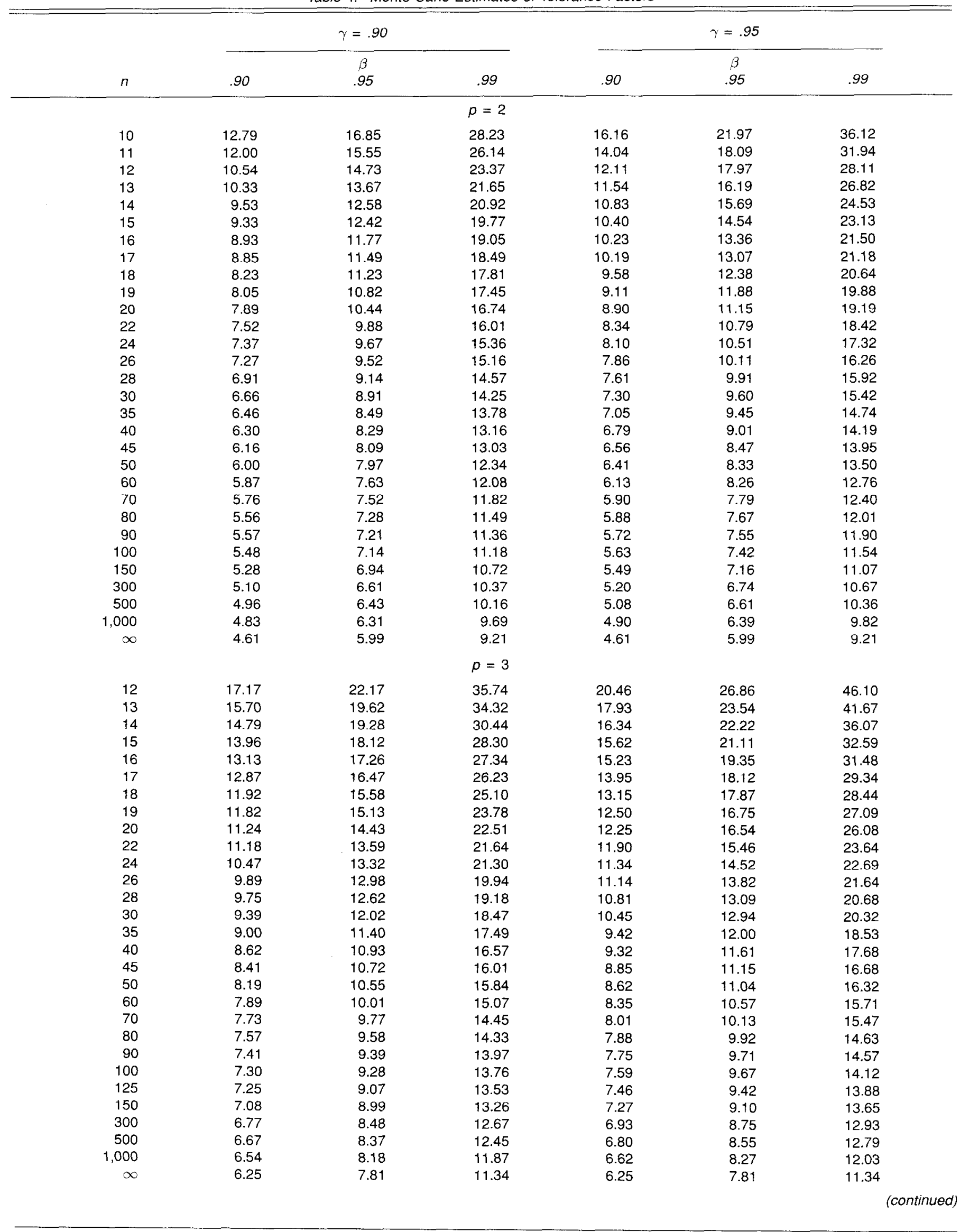


Table 4. (continued)

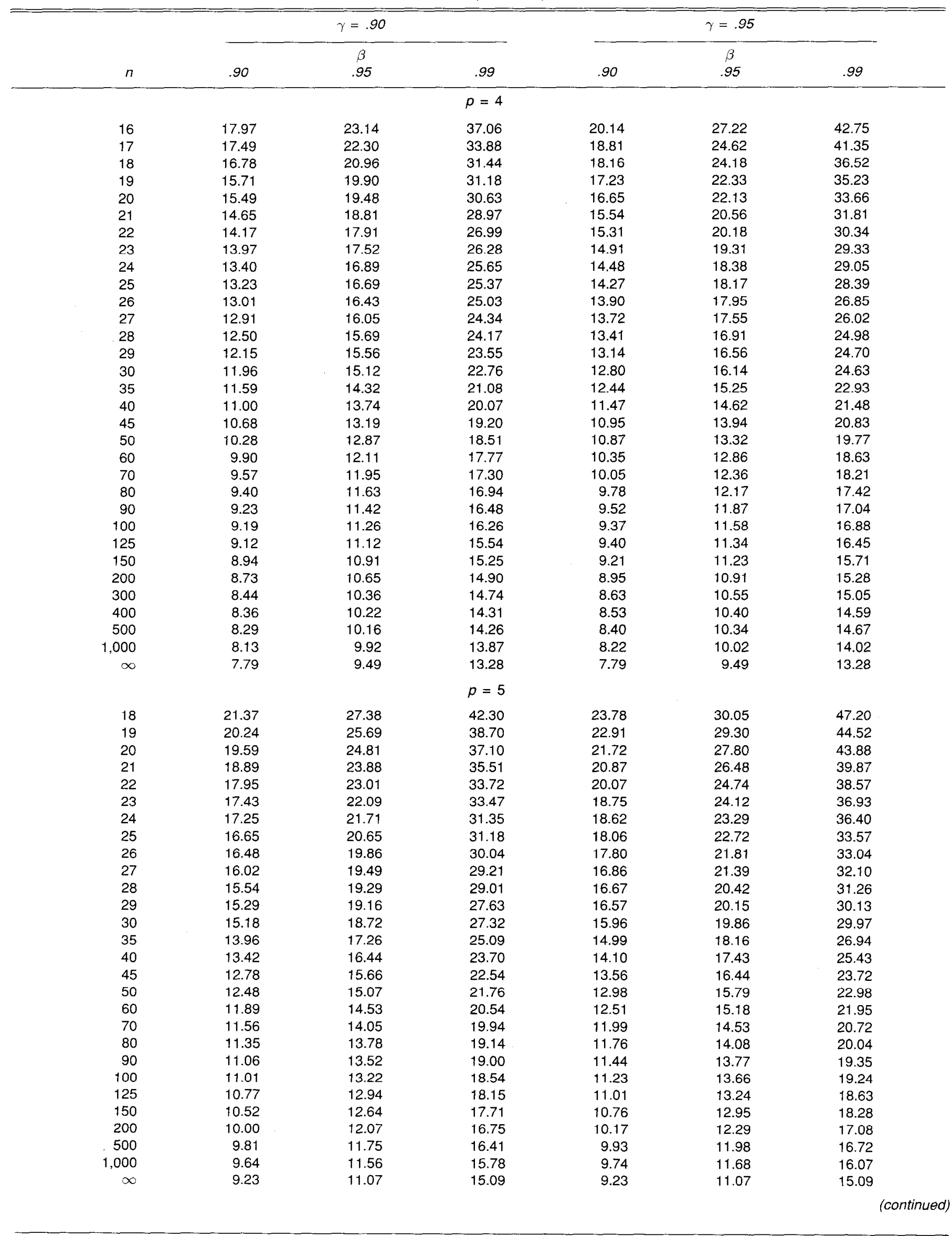


Table 4. (continued)

\begin{tabular}{|c|c|c|c|c|c|c|}
\hline \multirow[b]{3}{*}{$n$} & \multicolumn{3}{|c|}{$\gamma=.90$} & \multicolumn{3}{|c|}{$\gamma=.95$} \\
\hline & \multirow{3}{*}{\multicolumn{3}{|c|}{$\frac{.99}{p=6}$}} & \multicolumn{3}{|c|}{$\beta$} \\
\hline & & & & .90 & .95 & .99 \\
\hline & & & & & & \\
\hline 19 & 26.72 & 32.35 & 49.74 & 29.43 & 38.77 & 59.19 \\
\hline 20 & 24.86 & 32.00 & 46.19 & 27.22 & 35.11 & 54.09 \\
\hline 21 & 23.66 & 30.17 & 44.48 & 25.55 & 32.29 & 50.83 \\
\hline 22 & 22.27 & 28.39 & 42.98 & 24.09 & 31.46 & 48.63 \\
\hline 23 & 22.22 & 27.44 & 40.89 & 23.49 & 30.08 & 46.65 \\
\hline 24 & 21.25 & 26.77 & 38.82 & 23.34 & 27.98 & 43.25 \\
\hline 25 & 20.51 & 25.56 & 37.63 & 22.05 & 27.71 & 40.59 \\
\hline 26 & 20.01 & 24.64 & 36.17 & 21.37 & 26.81 & 40.01 \\
\hline 27 & 19.03 & 23.88 & 34.87 & 20.70 & 25.38 & 38.28 \\
\hline 28 & 18.75 & 23.32 & 34.38 & 19.87 & 25.15 & 37.79 \\
\hline 29 & 18.94 & 22.67 & 33.16 & 19.02 & 24.46 & 36.24 \\
\hline 30 & 18.15 & 22.28 & 32.45 & 19.62 & 24.08 & 36.16 \\
\hline 35 & 16.60 & 20.60 & 28.88 & 17.67 & 22.02 & 31.54 \\
\hline 40 & 15.81 & 19.17 & 27.32 & 16.97 & 20.43 & 29.10 \\
\hline 45 & 15.06 & 18.63 & 25.68 & 15.86 & 19.39 & 27.25 \\
\hline 50 & 14.72 & 17.87 & 24.91 & 15.34 & 18.71 & 26.27 \\
\hline 60 & 13.93 & 16.83 & 23.64 & 14.47 & 17.34 & 24.43 \\
\hline 70 & 13.41 & 16.31 & 22.68 & 13.96 & 16.78 & 23.43 \\
\hline 80 & 13.16 & 15.81 & 21.80 & 13.63 & 16.33 & 22.50 \\
\hline 90 & 12.93 & 15.44 & 21.21 & 13.29 & 15.69 & 21.94 \\
\hline 100 & 12.72 & 15.14 & 20.97 & 13.08 & 15.75 & 21.58 \\
\hline 125 & 12.45 & 14.83 & 20.33 & 12.72 & 15.18 & 20.99 \\
\hline 150 & 12.14 & 14.42 & 19.86 & 12.38 & 14.82 & 20.22 \\
\hline 200 & 11.95 & 14.17 & 18.95 & 12.26 & 14.43 & 19.40 \\
\hline 250 & 11.76 & 13.92 & 18.63 & 12.05 & 14.22 & 19.01 \\
\hline 300 & 11.48 & 13.69 & 18.73 & 11.71 & 13.88 & 18.98 \\
\hline 500 & 11.32 & 13.36 & 18.26 & 11.45 & 13.60 & 18.66 \\
\hline 1,000 & 11.11 & 13.15 & 17.55 & 11.23 & 13.28 & 17.68 \\
\hline \multirow[t]{2}{*}{$\infty$} & 10.64 & 12.59 & 16.81 & 10.64 & 12.59 & 16.81 \\
\hline & & & $p=7$ & & & \\
\hline 20 & 31.03 & 39.95 & 59.18 & 35.40 & 44.15 & 69.23 \\
\hline 21 & 29.70 & 36.76 & 55.72 & 32.55 & 41.23 & 65.02 \\
\hline 22 & 28.07 & 35.13 & 51.77 & 31.41 & 40.26 & 57.15 \\
\hline 23 & 26.90 & 32.72 & 50.22 & 28.76 & 36.72 & 55.22 \\
\hline 24 & 25.47 & 31.99 & 47.47 & 27.87 & 35.21 & 53.15 \\
\hline 25 & 24.70 & 30.53 & 44.44 & 27.02 & 33.56 & 49.71 \\
\hline 26 & 24.38 & 29.49 & 42.99 & 25.50 & 31.92 & 46.50 \\
\hline 27 & 23.31 & 28.75 & 41.32 & 25.30 & 31.70 & 45.83 \\
\hline 28 & 22.64 & 27.87 & 40.83 & 24.50 & 30.55 & 45.37 \\
\hline 29 & 21.98 & 27.39 & 39.79 & 23.62 & 29.19 & 42.83 \\
\hline 30 & 21.32 & 26.41 & 38.00 & 23.44 & 28.57 & 41.12 \\
\hline 35 & 19.68 & 24.09 & 34.44 & 20.74 & 25.57 & 36.84 \\
\hline 40 & 18.46 & 22.18 & 31.82 & 19.66 & 23.64 & 33.91 \\
\hline 45 & 17.53 & 21.58 & 29.72 & 18.62 & 22.13 & 31.80 \\
\hline 50 & 16.99 & 20.35 & 28.18 & 17.79 & 21.32 & 30.14 \\
\hline 60 & 16.03 & 19.26 & 26.26 & 16.58 & 19.98 & 27.73 \\
\hline 70 & 15.47 & 18.27 & 25.12 & 15.87 & 18.98 & 26.13 \\
\hline 80 & 15.02 & 17.93 & 24.36 & 15.45 & 18.34 & 25.28 \\
\hline 90 & 14.72 & 17.46 & 23.60 & 15.10 & 17.88 & 24.41 \\
\hline 100 & 14.43 & 17.05 & 23.25 & 14.79 & 17.58 & 23.86 \\
\hline 125 & 13.99 & 16.59 & 22.37 & 14.29 & 16.93 & 23.07 \\
\hline 150 & 13.71 & 16.16 & 21.81 & 13.94 & 16.47 & 22.30 \\
\hline 200 & 13.39 & 15.70 & 21.13 & 13.63 & 16.06 & 21.67 \\
\hline 300 & 13.04 & 15.28 & 20.50 & 13.17 & 15.56 & 21.01 \\
\hline 400 & 12.85 & 15.05 & 20.21 & 13.00 & 15.32 & 20.52 \\
\hline 500 & 12.73 & 14.98 & 19.98 & 12.90 & 15.16 & 20.29 \\
\hline 750 & 12.62 & 14.86 & 19.82 & 12.79 & 15.03 & 20.17 \\
\hline 1,000 & 12.55 & 14.71 & 19.68 & 12.62 & 14.91 & 19.92 \\
\hline \multirow[t]{2}{*}{$\infty$} & 12.02 & 14.07 & 18.48 & 12.02 & 14.07 & 18.48 \\
\hline & & & & & & \\
\hline
\end{tabular}


Table 4. (continued)

\begin{tabular}{|c|c|c|c|c|c|c|}
\hline \multirow[b]{2}{*}{$n$} & \multicolumn{3}{|c|}{$\gamma=.90$} & \multicolumn{3}{|c|}{$\gamma=.95$} \\
\hline & 90 & $\begin{array}{l}\beta \\
.95 \\
\end{array}$ & .99 & .90 & $\begin{array}{l}\beta \\
.95\end{array}$ & .99 \\
\hline \multicolumn{7}{|c|}{$p=8$} \\
\hline 30 & 25.30 & 31.18 & 43.78 & 27.10 & 33.04 & 46.91 \\
\hline 31 & 25.00 & 30.24 & 43.38 & 26.61 & 32.18 & 46.41 \\
\hline 32 & 24.63 & 29.21 & 41.95 & 25.73 & 31.55 & 44.03 \\
\hline 33 & 23.83 & 28.91 & 41.06 & 24.94 & 30.76 & 43.66 \\
\hline 34 & 23.37 & 28.28 & 40.50 & 24.66 & 30.40 & 43.37 \\
\hline 35 & 22.80 & 27.96 & 39.24 & 24.12 & 29.59 & 41.94 \\
\hline 40 & 21.24 & 25.33 & 35.73 & 22.22 & 27.02 & 38.08 \\
\hline 45 & 20.12 & 24.01 & 33.34 & 21.26 & 25.20 & 35.39 \\
\hline 50 & 19.46 & 23.05 & 31.52 & 20.17 & 23.99 & 33.28 \\
\hline 60 & 18.19 & 21.36 & 29.44 & 18.87 & 22.46 & 30.49 \\
\hline 70 & 17.46 & 20.43 & 27.86 & 18.01 & 21.41 & 28.92 \\
\hline 80 & 16.94 & 19.99 & 26.90 & 17.30 & 20.52 & 27.82 \\
\hline 90 & 16.51 & 19.48 & 25.90 & 16.88 & 20.06 & 26.86 \\
\hline 100 & 16.19 & 19.10 & 25.63 & 16.51 & 19.61 & 26.21 \\
\hline 120 & 15.78 & 18.48 & 24.52 & 16.10 & 18.82 & 25.30 \\
\hline 140 & 15.49 & 18.11 & 24.01 & 15.82 & 18.55 & 24.60 \\
\hline 160 & 15.22 & 17.72 & 23.61 & 15.57 & 18.03 & 24.03 \\
\hline 180 & 15.04 & 17.53 & 23.32 & 15.34 & 17.86 & 23.79 \\
\hline 200 & 14.90 & 17.48 & 22.90 & 15.17 & 17.71 & 23.63 \\
\hline 250 & 14.67 & 17.17 & 22.66 & 14.97 & 17.32 & 23.16 \\
\hline 300 & 14.51 & 16.87 & 22.32 & 14.67 & 17.17 & 22.67 \\
\hline 400 & 14.26 & 16.62 & 21.87 & 14.56 & 16.81 & 22.40 \\
\hline 500 & 14.21 & 16.49 & 21.69 & 14.32 & 16.68 & 22.26 \\
\hline 1,000 & 13.96 & 16.22 & 21.01 & 14.09 & 16.37 & 21.16 \\
\hline$\infty$ & 13.36 & 15.51 & 20.09 & 13.36 & 15.51 & 20.09 \\
\hline \multicolumn{7}{|c|}{$\rho=9$} \\
\hline 35 & 26.45 & 31.28 & 44.03 & 27.98 & 34.23 & 48.47 \\
\hline 37 & 25.39 & 30.67 & 42.49 & 26.57 & 32.58 & 45.32 \\
\hline 40 & 24.52 & 28.92 & 40.34 & 25.56 & 30.16 & 42.90 \\
\hline 43 & 23.30 & 28.16 & 38.42 & 24.33 & 29.28 & 39.62 \\
\hline 45 & 23.07 & 27.19 & 37.42 & 24.02 & 28.33 & 39.29 \\
\hline 50 & 21.73 & 25.72 & 35.20 & 22.49 & 26.72 & 37.07 \\
\hline 55 & 21.25 & 24.92 & 33.56 & 21.88 & 25.69 & 35.43 \\
\hline 60 & 20.29 & 24.24 & 32.48 & 21.12 & 24.79 & 33.89 \\
\hline 65 & 19.87 & 23.49 & 31.40 & 20.40 & 24.12 & 32.71 \\
\hline 70 & 19.62 & 23.07 & 30.71 & 20.11 & 23.30 & 32.08 \\
\hline 75 & 19.21 & 22.47 & 29.92 & 19.60 & 23.12 & 30.83 \\
\hline 80 & 18.88 & 22.03 & 29.29 & 19.26 & 22.69 & 30.29 \\
\hline 85 & 18.66 & 21.67 & 29.07 & 19.10 & 22.44 & 29.90 \\
\hline 90 & 18.39 & 21.45 & 28.65 & 18.84 & 21.94 & 29.39 \\
\hline 95 & 18.11 & 21.32 & 28.02 & 18.52 & 21.63 & 28.72 \\
\hline 100 & 18.04 & 21.02 & 27.93 & 18.41 & 21.48 & 28.65 \\
\hline 125 & 17.28 & 20.06 & 26.64 & 17.72 & 20.54 & 27.48 \\
\hline 150 & 16.95 & 19.77 & 25.85 & 17.32 & 19.93 & 26.43 \\
\hline 175 & 16.68 & 19.31 & 25.44 & 16.94 & 19.61 & 26.04 \\
\hline 200 & 16.45 & 19.05 & 24.92 & 16.70 & 19.39 & 25.47 \\
\hline 250 & 16.11 & 18.68 & 24.53 & 16.36 & 19.08 & 24.91 \\
\hline 300 & 16.00 & 18.51 & 24.14 & 16.11 & 18.71 & 24.62 \\
\hline 400 & 15.70 & 18.15 & 23.73 & 15.97 & 18.41 & 24.08 \\
\hline 500 & 15.59 & 18.05 & 23.47 & 15.82 & 18.27 & 23.94 \\
\hline 750 & 15.40 & 17.78 & 23.14 & 15.53 & 18.02 & 23.50 \\
\hline 1,000 & 15.34 & 17.68 & 22.64 & 15.49 & 17.85 & 22.86 \\
\hline$\infty$ & 14.68 & 16.92 & 21.67 & 14.68 & 16.92 & 21.67 \\
\hline
\end{tabular}

situation is particularly unsatisfactory for the case $p=6$. For example, when $p=6, n=30$, and $\beta=\gamma=.90$, the tolerance intervals based on the arithmetic mean, geometric mean, and harmonic mean have simulated confidence levels $\hat{\gamma}$ equal to $.159, .482$, and .803 . Among the three, the harmonic-mean approximation exhibits relatively better performance. The justification for the harmonic-mean ap- proximation, given in Section 3.3, may partly explain this. The modified harmonic-mean approximation shows a better performance compared to the harmonic-mean approximation perhaps due to the accuracy of the approximation. The tolerance factor based on the harmonic-mean approximation is also slightly less than the actual tolcrance factor, however, in most of the cases considered for simula- 
Table 4. (continued)

\begin{tabular}{|c|c|c|c|c|c|c|}
\hline \multirow[b]{3}{*}{$n$} & \multicolumn{3}{|c|}{$\gamma=.90$} & \multicolumn{3}{|c|}{$\gamma=.95$} \\
\hline & & $\beta$ & & & $\beta$ & \\
\hline & .90 & .95 & .99 & .90 & .95 & .99 \\
\hline \multicolumn{7}{|c|}{$p=10$} \\
\hline 40 & 27.66 & 32.87 & 45.59 & 28.94 & 34.01 & 48.03 \\
\hline 45 & 25.76 & 30.46 & 41.68 & 26.60 & 31.89 & 44.10 \\
\hline 50 & 24.76 & 28.77 & 38.93 & 25.48 & 29.93 & 40.77 \\
\hline 55 & 23.47 & 27.52 & 37.54 & 24.40 & 28.56 & 38.70 \\
\hline 60 & 22.71 & 26.77 & 35.42 & 23.51 & 27.24 & 37.10 \\
\hline 65 & 22.26 & 26.00 & 34.88 & 22.90 & 26.47 & 35.84 \\
\hline 70 & 21.63 & 25.07 & 33.38 & 22.16 & 25.95 & 34.41 \\
\hline 75 & 21.25 & 24.73 & 32.54 & 21.72 & 25.42 & 33.41 \\
\hline 80 & 20.85 & 24.27 & 31.95 & 21.34 & 24.78 & 33.22 \\
\hline 85 & 20.44 & 23.90 & 31.40 & 20.91 & 24.64 & 32.60 \\
\hline 90 & 20.41 & 23.56 & 31.16 & 20.62 & 24.02 & 31.71 \\
\hline 95 & 20.04 & 23.23 & 30.52 & 20.45 & 23.83 & 31.26 \\
\hline 100 & 19.85 & 22.88 & 30.22 & 20.15 & 23.45 & 31.22 \\
\hline 125 & 19.07 & 22.03 & 28.88 & 19.49 & 22.48 & 29.38 \\
\hline 150 & 18.57 & 21.43 & 27.86 & 18.92 & 21.78 & 28.55 \\
\hline 175 & 18.24 & 20.99 & 27.39 & 18.53 & 21.38 & 27.81 \\
\hline 200 & 17.94 & 20.70 & 27.00 & 18.30 & 20.97 & 27.36 \\
\hline 250 & 17.65 & 20.30 & 26.24 & 17.94 & 20.51 & 26.79 \\
\hline 300 & 17.46 & 20.05 & 25.90 & 17.59 & 20.29 & 26.28 \\
\hline 400 & 17.17 & 19.74 & 25.51 & 17.31 & 19.97 & 25.93 \\
\hline 500 & 16.98 & 19.45 & 25.19 & 17.11 & 19.68 & 25.52 \\
\hline 1,000 & 16.68 & 19.11 & 24.25 & 16.88 & 19.33 & 24.50 \\
\hline$\infty$ & 15.99 & 18.31 & 23.21 & 15.99 & 18.31 & 23.21 \\
\hline
\end{tabular}

tion. Overall, $c_{v}$ provides a conservative tolerance factor except when $\beta$ is large and $n$ is small (see the results for $\beta=.99, p=2$, and $n=10$ and 20). The tolerance factor $c_{\text {siot }}$ is even more conservative when $n$ is small (see the results for $p=2$ and $n=10$ ). The tolerance factor based on $c_{v \mathrm{~mm}}$ is extremely satisfactory except for the cases in which $n$ is small and $\beta$ is large $(\beta=.99)$. Our overall recommendation is to use $c_{v \mathrm{hm}}$ for $\beta=.90$ and .95 and to use $c_{v}$ when $\beta=.99$.

Note that these approximations provide tolerance factors having explicit analytic forms and hence are easily computed. The numerical results, however, indicate that their pcrformance is somewhat unsatisfactory when the $\beta$ and $\gamma$ get large. In this case, estimating the tolerance factor by Monte Carlo simulation is the best option for those who have ready access to modern computing environments. For $\beta=.90, .95, .99$, and $\gamma=.90, .95$, Table 4 (pp. 243-247) gives such estimated tolerance factors for selected values of $n$ and $p=2(1) 10$. All the Monte Carlo estimates are based on 1,200 $\times 1,200$ runs. For large $p$ and small $n$, Monte Carlo estimates of the tolerance factors based on 1,200 by 1,200 runs are not stable, so they are not reported. In such situations, more stable estimates can be obtained by increasing the number of simulation runs to at least $10,000 \times 10,000$.

\section{CONCLUDING REMARKS}

It is clear that tolerance factors can be estimated by Monte Carlo simulation. The required computations, however, can be quite time consuming, especially in higher dimensions. Thus, it is highly desirable to have good approximations. This article is an attempt to arrive at such approximations. We have accomplished three goals. First, we have provided a review of the limited results that are available for the construction of tolerance regions for a multivariate normal population. Second, we have suggested some new approximate tolerance factors. Finally, we have made a comparison of the different approximate tolerance factors. It appears that such a comparison has not been attempted before. Based on the numerical results, we have been able to make some recommendations on the choice of the approximate tolerance factors for practical use. Our numerical results show that some of the approximate tolerance factors currently in use are very inaccurate and should not be used. Our study also suggests the direction in which research should proceed to arrive at even better approximate tolerance factors. For example, it appears quite likely that a choice of $\xi(V)$ between $p / \operatorname{tr}\left(V^{-1}\right)$ and $v_{11.2}$ [different from that in (3.16)] will provide a good approximation.

Fuchs and Kenett (1987, 1988) argued that many statistical-process-control problems are multivariate in nature. Yet, tolerance regions have not been widely used in this context, unlike in the univariate case, perhaps due to the unavailability of practical solutions that are easy to compute. We believe that our article has successfully addressed this issue. Our hope is that it will make multivariate tolerance regions popular and more accessible to practitioners.

\section{ACKNOWLEDGMENTS}

We are grateful to a referee, an associate editor, and Editor Max D. Morris for several helpful suggestions. The second author's research was supported in part by National Science Foundation Grant DMS 95-30932. 


\section{APPENDIX A: PROOF OF THEOREM 2.1}

Let $\mathbf{y}=\Sigma^{-1 / 2}(\mathbf{x}-\mu), \mathbf{u}=\Sigma^{-1 / 2}(\overline{\mathbf{x}}-\mu)$, and $V=$ $\Sigma^{-1 / 2} A \Sigma^{-1 / 2}$. Note that $\mathbf{y}, \mathbf{u}$, and $V$ are independent with $\mathbf{y} \sim N_{p}\left(0, I_{p}\right), \mathbf{u} \sim N_{p}\left(0,(1 / n) I_{p}\right)$, and $V \sim W_{p}\left(I_{p}, n-1\right)$, In terms of these variables, (2.3) can be expressed as

$$
P_{\mathbf{u}, V}\left[P_{\mathbf{y}}\left[(n-1)(\mathbf{y}-\mathbf{u})^{\prime} V^{-1}(\mathbf{y}-\mathbf{u}) \leq c \mid \mathbf{u}, V\right] \geq \beta\right]=\gamma .
$$

Let $\Gamma$ be a $p \times p$ orthogonal matrix such that $\Gamma^{\prime} V \Gamma=$ $\operatorname{diag}\left(l_{1}, \ldots, l_{p}\right)$, where $l_{i}$ 's are the eigenvalues of $V$. We can assume that $l_{1}>\cdots>l_{p}>0$. Let $\mathbf{z}=\Gamma^{\prime} \mathbf{y}$ and $\mathbf{q}=\Gamma^{\prime} \mathbf{u}$. Using this transformation, we can write (A.1) as

$$
P_{\mathbf{q}, \mathbf{l}}\left[P_{\mathbf{z}}\left[(n-1) \sum_{i=1}^{p}\left(z_{i}-q_{i}\right)^{2} / l_{i} \leq c \mid \mathbf{q}, \mathbf{l}\right] \geq \beta\right]=\gamma
$$

where $\mathbf{z}=\left(z_{1}, \ldots, z_{p}\right)^{\prime}, \mathbf{q}=\left(q_{1}, \ldots, q_{p}\right)^{\prime}$, and $\mathbf{l}=$ $\left(l_{1}, l_{2}, \ldots, l_{p}\right)^{\prime}$. The conditional distribution of $\mathbf{z}$, given $V$ and $\mathbf{q}$, is $N_{p}\left(0, I_{p}\right)$, and hence conditionally given $V$ and q, $\left(z_{i}-q_{i}\right)^{2} \sim \chi^{2}\left(1, q_{i}^{2}\right)$, and are independent for $i=$ $1, \ldots, p$, where $\chi^{2}(m, \delta)$ denotes the noncentral chi-squared random variable with $\mathrm{df}=m$ and noncentrality parameter $\delta$. It is difficult to derive the exact conditional distribution in (A.2), and hence John (1963) suggested the following approximation.

Let $\xi(\mathrm{l})$ be a real-valued function of $\mathbf{l}=\left(l_{1}, l_{2}, \ldots, l_{p}\right)^{\prime}$ such that $l_{p} \leq \xi(\mathbf{l}) \leq l_{1}$. Because $l_{1}>\cdots>l_{p}>0$, we have

$$
\begin{aligned}
& P_{\mathbf{z}}\left[(n-1) \sum_{i=1}^{p} \frac{\left(z_{i}-q_{i}\right)^{2}}{l_{p}} \leq c \mid \mathbf{q}, \mathbf{l}\right] \\
& \quad \leq P_{\mathbf{z}}\left[(n-1) \sum_{i=1}^{p} \frac{\left(z_{i}-q_{i}\right)^{2}}{l_{i}} \leq c \mid \mathbf{q}, \mathbf{l}\right] \\
& \quad \leq P_{\mathbf{z}}\left[(n-1) \sum_{i=1}^{p} \frac{\left(z_{i}-q_{i}\right)^{2}}{l_{1}} \leq c \mid \mathbf{q}, \mathbf{1}\right]
\end{aligned}
$$

and

$$
\begin{aligned}
& P_{\mathbf{z}}\left[(n-1) \sum_{i=1}^{p} \frac{\left(z_{i}-q_{i}\right)^{2}}{l_{p}} \leq c \mid \mathbf{q}, 1\right] \\
& \quad \leq P_{\mathbf{z}}\left[(n-1) \sum_{i=1}^{p} \frac{\left(z_{i}-q_{i}\right)^{2}}{\xi(\mathbf{l})} \leq c \mid \mathbf{q}, 1\right] \\
& \quad \leq P_{\mathbf{z}}\left[(n-1) \sum_{i=1}^{p} \frac{\left(z_{i}-q_{i}\right)^{2}}{l_{1}} \leq c \mid \mathbf{q}, \mathbf{l}\right] .
\end{aligned}
$$

Furthermore, John (1963) asserted that, for large $n$,

$$
\begin{aligned}
P_{\mathbf{z}}[(n-1) & \left.\sum_{i=1}^{p}\left(z_{i}-q_{i}\right)^{2} / l_{1} \leq c \mid \mathbf{q}, \mathbf{l}\right] \\
& \doteq P_{\mathbf{z}}\left[(n-1) \sum_{i=1}^{p}\left(z_{i}-q_{i}\right)^{2} / l_{p} \leq c \mid \mathbf{q}, \mathbf{1}\right] .
\end{aligned}
$$

Thus, for large $n$, it follows from (A.3), (A.4), and ( $\Lambda .5$ ) that

$$
\begin{aligned}
& P_{\mathbf{z}}\left[(n-1) \sum_{i=1}^{p}\left(z_{i}-q_{i}\right)^{2} / l_{i} \leq c \mid \mathbf{q}, 1\right] \\
& \quad \doteq P_{\mathbf{z}}\left[(n-1) \sum_{i=1}^{p}\left(z_{i}-q_{i}\right)^{2} / \xi(\mathbf{l}) \leq c \mid \mathbf{q}, 1\right] .
\end{aligned}
$$

Substituting the right side of (A.6) in (A.2), we want to compute $c$ satisfying

$$
\left.P_{\mathbf{q}, \mathbf{l}}\left[P_{\mathbf{z}}\left[(n-1) \sum_{i=1}^{p}\left(z_{i}-q_{i}\right)^{2} / \xi(\mathbf{l}) \leq c \mid \mathbf{q}, \mathbf{l}\right] \geq \beta\right]\right]=\gamma
$$

or, equivalently,

$$
\left.P_{\mathbf{q}, \mathbf{l}}\left[P_{\mathbf{z}}\left[\sum_{i=1}^{p}\left(z_{i}-q_{i}\right)^{2} \leq \frac{c \xi(\mathbf{l})}{n-1} \mid \mathbf{q}, \mathbf{l}\right] \geq \beta\right]\right]=\gamma .
$$

Because, given $\mathbf{q}, \sum_{i=1}^{p}\left(z_{i}-q_{i}\right)^{2} \sim \chi^{2}\left(p, \sum_{i=1}^{p} q_{i}^{2}\right)$, (A.7) is equivalent to

$$
P_{\mathbf{q}, 1}\left[\frac{c \xi(\mathbf{l})}{n-1} \geq \chi^{2}\left(\beta ; p, \sum_{i=1}^{p} q_{i}^{2}\right)\right]=\gamma .
$$

We note that $\sum_{i=1}^{p} q_{i}^{2}=\mathbf{q}^{\prime} \mathbf{q}=\mathbf{u}^{\prime} \mathbf{u}$ and $n \mathbf{u}^{\prime} \mathbf{u} \sim \chi^{2}(p)$. Hence, $E\left(\sum_{i=1}^{p} q_{i}^{2}\right)=p / n$. As a further approximation, John (1963) replaced $\chi^{2}\left(\beta ; p, \sum_{i=1}^{p} q_{i}^{2}\right)$ by $\chi^{2}(\beta ; p, p / n)$ and justifies it for large $n$. With this approximation, (A.8) becomes

$$
P_{\mathbf{1}}\left[\frac{c \xi(1)}{n-1} \geq \chi^{2}(\beta ; p, p / N)\right]=\gamma
$$

It is clear that (A.9) is equivalent to (2.5).

\section{APPENDIX B: THE FIRST TWO MOMENTS OF $\left\{\left[\operatorname{tr}\left(V^{-1}\right) / p\right]+V_{11.2}^{-1}\right\} / 2$}

Let

$$
W=\left[\frac{\operatorname{tr}\left(V^{-1}\right)}{p}+v_{11.2}^{-1}\right] / 2
$$

and consider the partitioning given in (3.14). Recall that $V \sim W_{p}\left(I_{p}, n-1\right)$, the $p$-dimensional Wishart distribution with $\mathrm{df}=n-1$ and associated scale matrix $I_{p}$. We shall use some of the following properties of the Wishart distribution for the matrix $V$ in terms of the partitioning given in (3.14) [for a proof of these properties, we refer to Muirhead (1982, theorem 3.2.10)]:

1. $v_{11.2} \sim \chi^{2}(n-p)$.

2. $V_{22}^{-1 / 2} \mathbf{v}_{21} \sim N\left(\mathbf{0}, I_{p-1}\right)$.

3. $V_{22} \sim W_{p-1}\left(I_{p-1}, n-1\right)$.

4. $v_{11.2}, V_{22}^{-1 / 2} \mathbf{v}_{21}$, and $V_{22}$ are independently distributed. 
The following expected values can be easily derived using the distributional results in (B.2); see also (3.9):

$$
\begin{aligned}
& E\left(v_{11.2}^{-1}\right)=1 /(n-p-2) \\
& E\left(v_{11.2}^{-2}\right)=\frac{1}{(n-p-2)(n-p-4)} \\
& E\left[\operatorname{tr}\left(V^{-1}\right)\right]=p /(n-p-2) \\
& E\left[\left\{\operatorname{tr}\left(V^{-1}\right)\right\}^{2}\right]=\frac{p[(n-p-3) p+2]}{(n-p-1)(n-p-2)(n-p-4)} \\
& E\left[\operatorname{tr}\left(V_{22}^{-1}\right)\right]=(p-1) /(n-p-1) \\
& E\left(V_{22}^{-1 / 2} \mathbf{v}_{21} \mathbf{v}_{12} V_{22}^{-1 / 2}\right)=I_{p-1} .
\end{aligned}
$$

Using (B.1) and (B.3) and simplifying, we get

$$
E(W)=1 /(n-p-2) .
$$

Moreover,

$$
\begin{aligned}
E\left(W^{2}\right)=\frac{1}{4} & {\left[\frac{1}{p^{2}} E\left\{\left(\operatorname{tr}\left(V^{-1}\right)\right)^{2}\right\}+E\left(v_{11.2}^{-2}\right)\right.} \\
& \left.\quad+\frac{2}{p} E\left\{v_{11.2}^{-1} \operatorname{tr}\left(V^{-1}\right)\right\}\right] \\
=\frac{1}{4}[ & \frac{[(n-p-3) p+2]}{p(n-p-1)(n-p-2)(n-p-4)} \\
& +\frac{1}{(n-p-2)(n-p-4)} \\
& \left.+\frac{2}{p} E\left\{v_{11.2}^{-1} \operatorname{tr}\left(V^{-1}\right)\right\}\right]
\end{aligned}
$$

Using the expression for the inverse of a partitioned matrix (see Rao 1973, p. 33), we get

$$
\begin{aligned}
\operatorname{tr}\left(V^{-1}\right)= & v_{11.2}^{-1}+\operatorname{tr}\left(V_{22}^{-1}\right) \\
& +v_{11.2}^{-1} \operatorname{tr}\left[V_{22}^{-1} \mathbf{v}_{21} \mathbf{v}_{12} V_{22}^{-1}\right] \\
= & v_{11.2}^{-1}+\operatorname{tr}\left(V_{22}^{-1}\right) \\
& +v_{11.2}^{-1} \operatorname{tr}\left[\left(V_{22}^{-1 / 2} \mathbf{v}_{21} \mathbf{v}_{12} V_{22}^{-1 / 2}\right) V_{22}^{-1}\right]
\end{aligned}
$$

Using (B.6), the last term in (B.5) can be written as

$$
\begin{aligned}
& E\left\{v_{11.2}^{-1} \operatorname{tr}\left(V^{-1}\right)\right\}=E\left(v_{11.2}^{-2}\right)+E\left(v_{11.2}^{-1}\right) E\left[\operatorname{tr}\left(V_{22}^{-1}\right)\right] \\
& +E\left(v_{11.2}^{-2}\right) \operatorname{tr}\left[E\left(V_{22}^{-1 / 2} \mathbf{v}_{21} \mathbf{v}_{12} V_{22}^{-1 / 2}\right) E\left(V_{22}^{-1}\right)\right],
\end{aligned}
$$

where we have used the independence of $v_{11.2}, V_{22}^{-1 / 2} \mathbf{v}_{21}$, and $V_{22}$. The expected values required to simplify (B.7) are given in (B.3). On simplification, we get

$$
E\left(v_{11.2}^{-1} \operatorname{lr}\left(V^{-1}\right)\right)=\frac{(n-p-1)+(n-p-3)(p-1)}{(n-p-1)(n-p-2)(n-p-4)}
$$

Substituting (B.8) into (B.5) and simplifying, we get

$$
E\left(W^{2}\right)=\frac{2 p(n-p-1)-3(p-1)}{2 p(n-p-1)(n-p-2)(n-p-4)}
$$

\section{[Received March 1998. Revised February 1999.]}

\section{REFERENCES}

Anderson, T. W. (1984), An Introduction to Multivariate Statistical Analysis (2nd ed.), New York: Wiley.

Chew, V. (1966), "Confidence, Prediction, and Tolerance Regions for the Multivariate Normal Distribution," Journal of the American Statistical Association, 61, 605-617.

Eberhardt, K. R., Mee, R. W., and Reeve, C. P. (1989), "Computing Factors for Exact Two-Sided Tolerance Intervals for a Normal Distribulion," Communications in Statistics-Simulation and Computation, 18, 397-413.

Fuchs, C., and Kenett, R. S. (1987), "Multivariate Tolerance Regions and F-Tests," Journal of Quality Technology, 19, 122-131.

(1988), "Appraisal of Ceramic Substrates by Multivariate Tolerance Regions," The Statistician, 37, 401-411.

Guttman, I. (1970a), Statistical Tolerance Regions, Classical and Bayesian, London: Griffin's Statistical Monographs.

$-(1970 \mathrm{~b})$, "Construction of $\beta$-Content Tolerance Regions at Confidence Level $\gamma$ for Large Samples From the $k$-Variate Normal Distribution," The Annals of Mathematical Statistics, 41, 376-400.

Hall, I. J., and Sheldon, D. D. (1979), "Improved Bivariate Normal Tolerance Regions With Some Applications," Journal of Quality Technology, $11,13-19$.

Hoel, P. G. (1937), "A Significance Test for Component Analysis," The Annals of Mathematical Statistics, 8, 149-158.

John, S. (1963), "A Tolerance Region for Multivariate Normal Distributions," Sankhyā, Ser. A, 25, 363-368.

Kendall, M. G., and Stuart, A. (1973), The Advanced Theory of Statistics, Vol. 2, New York: Hafner.

Muirhead, R. J. (1982), Aspects of Multivariate Statistical Theory, New York: Wiley.

Rao, C. R. (1973), Linear Statistical Inference and Its Applications (2nd ed.), New York: Wiley.

Siotani, M. (1964), "Tolerance Regions for a Multivariate Normal Population," Annals of the Institute of Statistical Mathematics, 16, 135-153.

Smith, W. B., and Hocking, R. R. (1972), "Wishart Variates Generator (algurithm AS 53)," Applied Statistics, 21, 341-345.

von Rosen, D. (1988), "Moments for the Inverted Wishart Distribution," Scandinavian Journal of Statistics, 15, 97-109.

Wald, A. (1942), "Setting of Tolerance Limits When the Sample is Large," The Annals of Mathematical Statistics, 13, 389-399.

Wald, A., and Wolfowitz, J. (1946), "Tolerance Limits for a Normal Distribution," The Annals of Mathematical Statistics, 17, 208-215. 